\title{
Properties of Magnetohydrodynamic Modes in Compressively Driven Plasma Turbulence
}

\author{
K. D. Makwana ${ }^{1, *}$ and Huirong Yan $\circledast^{1,2, \dagger}$ \\ ${ }^{1}$ Deutsches Elektronen Synchrotron (DESY), Platanenallee 6, D-15738 Zeuthen, Germany \\ ${ }^{2}$ Institut für Physik und Astronomie, Universität Potsdam, D-14476 Potsdam, Germany
}

(Received 4 July 2019; revised 15 May 2020; accepted 27 May 2020; published 28 July 2020)

\begin{abstract}
We study properties of magnetohydrodynamic (MHD) eigenmodes by decomposing the data of MHD simulations into linear MHD modes-namely, the Alfvén, slow magnetosonic, and fast magnetosonic modes. We drive turbulence with a mixture of solenoidal and compressive driving while varying the Alfvén Mach number $\left(M_{A}\right)$, plasma $\beta$, and the sonic Mach number from subsonic to transsonic. We find that the proportion of fast and slow modes in the mode mixture increases with increasing compressive forcing. This proportion of the magnetosonic modes can also become the dominant fraction in the mode mixture. The anisotropy of the modes is analyzed by means of their structure functions. The Alfvén-mode anisotropy is consistent with the Goldreich-Sridhar theory. We find a transition from weak to strong Alfvénic turbulence as we go from low to high $M_{A}$. The slow-mode properties are similar to the Alfvén mode. On the other hand, the isotropic nature of fast modes is verified in the cases where the fast mode is a significant fraction of the mode mixture. The fast-mode behavior does not show any transition in going from low to high $M_{A}$. We find indications that there is some interaction between the different modes, and the properties of the dominant mode can affect the properties of the weaker modes. This work identifies the conditions under which magnetosonic modes can be a major fraction of turbulent astrophysical plasmas, including the regime of weak turbulence. Important astrophysical implications for cosmic-ray transport and magnetic reconnection are discussed.
\end{abstract}

DOI: 10.1103/PhysRevX.10.031021

Subject Areas: Astrophysics, Plasma Physics

\section{INTRODUCTION}

Plasma turbulence plays an important role in various astrophysical processes. It is important in solar wind heating and acceleration [1], it regulates star formation processes [2-4], and it scatters cosmic rays [5], to name a few. The properties of turbulence depend on the underlying modes it is made up of. The magnetohydrodynamic (MHD) system of equations of a 3D, homogeneous, uniform, isothermal plasma with a uniform background magnetic field allows for three separate propagating linear eigenmodesthe Alfvén mode [6], the slow magnetosonic mode, and the fast magnetosonic mode [7]. Alfvénic turbulence (turbulence consisting of mostly Alfvén modes interacting with each other) is thought to be quite important in solar turbulence as Alfvén waves have been observed in solar wind [8]. Alfvénic turbulence has been studied for several decades, and several theories have been developed to

\footnotetext{
*kirit.makwana@desy.de

†huirong.yan@desy.de
}

Published by the American Physical Society under the terms of the Creative Commons Attribution 4.0 International license. Further distribution of this work must maintain attribution to the author(s) and the published article's title, journal citation, and DOI. describe it. The Alfvén modes are incompressible solutions to the linearized MHD equations. In the regime of strong turbulence, a critical balance is conjectured to be reached between the linear interaction time of wave packets and their nonlinear cascade time. As a result, scale-dependent anisotropy appears [9]. In the limit of weak turbulence, the resonant three-wave couplings involve only the nonpropagating Alfvén modes and produce a cascade in the wave vectors perpendicular to the local mean magnetic field direction only [10].

There has not been a comprehensive theory of turbulence consisting of the compressible MHD modes, namely, the slow and fast magnetosonic modes. Turbulence in the interstellar medium is identified by the measurement of density fluctuations in it, indicating the presence of compressible turbulence [11]. These turbulent density fluctuations in the interstellar medium molecular clouds are closely linked to star formation [12-14]. Numerical simulations of compressible turbulence to identify the inertial range scalings are more difficult and complex, with varying results depending on the plasma parameters, like Mach number [15-17]. Since astrophysical turbulence is expected to be magnetized and compressible, the magnetosonic modes should be considered when studying such turbulence. Whether the cascades of these different MHD modes are independent of each other and what their 
nature is are still open questions. The slow modes are cascaded by the shear-Alfvén modes and hence are expected to behave like the Alfvén modes [18]. Earlier studies indicated that the interactions between the Alfvén and the magnetosonic modes are limited on scales smaller than the injection scale. They also have shown that the energy spectrum and anisotropy of slow modes are quite similar to Alfvén modes [19]. On the other hand, fast modes have been seen to be quite different in their spectrum and anisotropy characteristics. Unlike Alfvén modes, which preferentially cascade in the field-perpendicular direction, fast modes seem to show an isotropic cascade. This result has also led to several important implications for astrophysical turbulence. Based on this result, it has been shown that fast modes could be the most effective scatterers of cosmic-ray particles [20,21].

Particle scattering and diffusion critically depend on the properties of plasma turbulence. While fast modes can play an important role in scattering of cosmic rays, simulations have shown that the fast modes might only be a marginal component of compressible turbulence [22]. However, these simulations have been driven incompressively by solenoidal forcing [15,19,22-24]. Thus, a natural question to ask is whether and how the nature of the forcing affects the mode composition of turbulence. We try to answer this question by compressively driving turbulence in a variety of different plasma parameter regimes. Some earlier studies have driven turbulence by keeping a mixture of solenoidal and compressive velocity fields at large scales [25] or by decomposing the driving force into solenoidal and compressive components [26]. We adopt a similar forcing but focus on the MHD mode decomposition. We find that the nature of the forcing significantly affects the composition of the turbulence in terms of the MHD modes. Another phenomenon less explored in numerical simulations is low$M_{A}$ (ratio of rms velocity to Alfvén speed) turbulence. Theoretically, the Alfvénic turbulent cascade is expected to be weak up to some scale and then transition to a state of strong turbulence, mediated by the critical balance condition, at smaller scales. This transition from weak to strong turbulence has only recently been simulated in decaying turbulence [27]. We explore the $M_{A}$ dependence of this transition in our compressively driven simulations and find that the nature of Alfvénic turbulence can significantly change with $M_{A}$.

The isotropic cascade properties of the fast mode have been studied at limited resolution previously [19], and the spectrum of this cascade was cautiously claimed to be $k^{-3 / 2}$. Higher-resolution studies are needed to verify this behavior through a well-resolved inertial range. We perform higher-resolution studies and find the isotropic nature of the fast modes throughout the inertial range, suggesting no scale-dependent anisotropy. Another related question is whether fast modes also show an $M_{A}$-dependent behavior like Alfvén modes in terms of weak or strong turbulence.
We do not find any such dependence for the fast modes. Our results also suggest that these different modes that cascade are not completely independent of each other, depending on which mode is dominant. This study shows that the nature of MHD turbulence can be different depending on a variety of parameters, particularly, the nature of driving, and this has important implications for understanding the effect of this turbulence on related problems.

\section{SIMULATION SETUP AND MODE DECOMPOSITION}

The simulations are performed by using the PLUTO code [28]. The ideal MHD equations are solved with no explicit resistivity or viscosity, only with numerical dissipation. The isothermal equation of state is used. The built-in HLLD Riemann solver [29] is utilized in conjunction with a WENO3 reconstruction scheme [30]. The time stepping is done by a third-order Runge-Kutta scheme. The simulation box is a cube of length $L_{x}=L_{y}=L_{z}=1$. The normalization is such that the Alfvén velocity $v_{A}$ and the mean magnetic field $B_{0}$ are numerically same. The mean field $\boldsymbol{B}_{0}$ is in the $z$ direction, which is the global parallel direction. The dimensionless quantities, like plasma $\beta$ and Mach numbers, are given in Table I. The sound speed $\left(c_{s}\right)$ is changed to vary the plasma $\beta$ defined as $\beta \equiv 2\left(c_{s} / v_{A}\right)^{2}$.

Turbulence is driven by using a readily available forcing module in PLUTO. This method drives turbulence by adding a force $\boldsymbol{F}^{\text {turb }}$ in both the momentum and energy equations. This force is modeled as an Ornstein-Uhlenbeck (OU)

TABLE I. Simulation parameters in a steady state with simulation IDs. The energy injection rate $E_{\text {inj }}$, the plasma $\beta$, Alfvén Mach number $M_{A}$, sonic Mach number $M_{S}$, the forcing correlation time $T$, resolution, and fraction of compressive driving $\zeta$ are varied amongst the different simulation runs.

\begin{tabular}{lccccccc}
\hline \hline ID & $E_{\text {inj }}$ & $\beta$ & $M_{A}$ & $M_{S}$ & T & Resolution & $\zeta$ \\
\hline S1a & $10^{-8}$ & 2.17 & 0.24 & 0.23 & 20 & $512^{3}$ & 1.0 \\
S2a & $8 \times 10^{-8}$ & 2.17 & 0.46 & 0.44 & 10 & $512^{3}$ & 1.0 \\
S2.5a & $2 \times 10^{-7}$ & 2.17 & 0.59 & 0.56 & 8.5 & $512^{3}$ & 1.0 \\
S3a & $5 \times 10^{-7}$ & 2.17 & 0.69 & 0.66 & 7.5 & $512^{3}$ & 1.0 \\
S4a & $8 \times 10^{-6}$ & 2.17 & 0.99 & 0.95 & 5 & $512^{3}$ & 1.0 \\
C1a & $3 \times 10^{-7}$ & 2.17 & 0.22 & 0.21 & 20 & $512^{3}$ & 0.1 \\
C2a & $5 \times 10^{-6}$ & 2.17 & 0.48 & 0.46 & 10 & $512^{3}$ & 0.1 \\
C3a & $2 \times 10^{-5}$ & 2.17 & 0.66 & 0.63 & 7.5 & $512^{3}$ & 0.1 \\
C4a & $9 \times 10^{-5}$ & 2.17 & 1.03 & 0.99 & 5 & $512^{3}$ & 0.1 \\
CB0a & $8 \times 10^{-6}$ & 0.5 & 0.51 & 1.02 & 10 & $512^{3}$ & 0.1 \\
CB1a & $3 \times 10^{-6}$ & 8.0 & 0.60 & 0.3 & 10 & $512^{3}$ & 0.1 \\
S1b & $10^{-8}$ & 2.17 & 0.25 & 0.24 & 20 & $1024^{3}$ & 1.0 \\
S2b & $8 \times 10^{-8}$ & 2.17 & 0.48 & 0.46 & 10 & $1024^{3}$ & 1.0 \\
S3b & $5 \times 10^{-7}$ & 2.17 & 0.72 & 0.69 & 7.5 & $1024^{3}$ & 1.0 \\
S4b & $8 \times 10^{-6}$ & 2.17 & 0.87 & 0.84 & 5 & $1024^{3}$ & 1.0 \\
C1b & $3 \times 10^{-7}$ & 2.17 & 0.23 & 0.22 & 20 & $1024^{3}$ & 0.1 \\
C4b & $9 \times 10^{-5}$ & 2.17 & 1.05 & 1.01 & 5 & $1024^{3}$ & 0.1 \\
\hline \hline
\end{tabular}


process $[31,32]$. This process is a stochastic differential equation governing the evolution of the force $\boldsymbol{F}^{\text {turb }}$, given by

$d \boldsymbol{F}^{\mathrm{turb}}(\boldsymbol{k}, t)=\boldsymbol{F}_{0}^{\mathrm{turb}}(\boldsymbol{k}) \mathcal{P}^{\zeta}(\boldsymbol{k}) d \mathcal{W}(t)-\boldsymbol{F}^{\mathrm{turb}}(\boldsymbol{k}, t) \frac{d t}{T}$.

Here, $d \boldsymbol{F}$ is a force added at every time step to the existing force $\boldsymbol{F}^{\mathrm{turb}}(\boldsymbol{k})$ at wave vector $\boldsymbol{k}$. The $\mathcal{P}^{\zeta}(\boldsymbol{k})$ operator is a projection operator, which separates the solenoidal and compressive parts of the force,

$\mathcal{P}_{i j}^{\zeta}(\boldsymbol{k})=\zeta \mathcal{P}_{i j}^{\perp}(\boldsymbol{k})+(1-\zeta) \mathcal{P}_{i j}^{\|}(\boldsymbol{k})=\zeta \delta_{i j}+(1-2 \zeta) \frac{k_{i} k_{j}}{k^{2}}$.

When $\zeta=1$, the forcing is purely solenoidal; when $\zeta=0$, it is purely compressive, and intermediate values give a mixture of solenoidal and compressive forcing. The forcing is limited to a range of wave numbers $k_{\min } \leq k \leq k_{\max }$. We ignore the $2 \pi$ factor in the definition of the wave number, and since the box length is also normalized to unity, the smallest wave number possible in our simulation is 1 . Thus, $k_{\min }=1$ and $k_{\max }=3$ in our simulations. We vary the Mach number $M_{A}$ of the turbulence by varying the energy injected into the turbulence $E_{\text {inj. }}$. We also vary the plasma $\beta$ by changing the isothermal sound speed $c_{s}$. This process also changes the sonic Mach number as shown in Table I for the different simulations. We scan the sonic Mach number from the subsonic to the transsonic range. We utilize solenoidal driving $(\zeta=1)$ and mostly compressive driving $(\zeta=0.1)$. Two different grid resolutions are utilized, $512^{3}$ and $1024^{3}$. Table I shows the different simulations we have analyzed and their parameters. The first letter "S" or "C" in the simulation ID represents whether it is solenoidally or compressively driven. The letter "a" or " $b$ " denotes the resolution $\left(512^{3}\right.$ or $1024^{3}$, respectively). Table I also lists the correlation time of the forcing $T$. This time is close to the usual value of the eddy turnover time at the injection scale $\left(L_{\text {inj }}\right), T \approx\left(L_{\text {inj }}\right) / v \approx$ $(1 / 2) /\left(v_{A} M_{A}\right)$ [33]. The simulations are run for several tens of eddy correlation time $T$ so that there are many snapshots of a statistical steady state.

The mode decomposition is a linear eigenmode decomposition, where the MHD state vector comprised of the density, velocity, and magnetic fluctuations is decomposed into a linear combination of the two Alfvén-mode eigenvectors, the two fast magnetosonic-mode eigenvectors and the two slow magnetosonic-mode eigenvectors. The MHD description is generally regarded as valid on scales much larger than the typical kinetic scales, like mean free path, ion skin depth, and gyroradius. Although kinetic damping can affect the compressible modes on collisionless scales [34,35], in typical warm ISM plasmas there is a vast range of scales from the injection (a few tens to $100 \mathrm{pcs}$ ) to collisionless scales such that the MHD prescription is justified for a large range of scales. The linear MHD mode decomposition assumes a homogeneous plasma without strong gradients. It is applicable if the turbulence driving scale is less than or equal to the scale length of the gradients. The mode decomposition is valid in those regions where the background plasma is devoid of strong gradients or discontinuities and where the driving can also be considered homogeneous. In situ observations of the solar wind have revealed the presence of the Alfvén and slow modes [36]. If we consider the intragalactic media with a scale height of a few hundred pc, then regions of size less than or similar to $100 \mathrm{pc}$ would be homogeneous in the absence of strong discontinuities. Many observations also indicate that turbulence is driven on galactic scales with an injection scale of about $100 \mathrm{pc}$ [37]. In such regions with sizes smaller than the injection scales, the turbulence driving can be considered homogeneous and the linear MHD mode analysis should apply.

We follow the prescription of Ref. [19] to decompose the MHD data into the MHD eigenmodes. The sonic and Alfvén Mach numbers are kept less than or close to 1 so that the nonlinear terms $\left(\delta \boldsymbol{v}^{2}, \delta \boldsymbol{b}^{2}\right)$ are not stronger than the linear terms $\left(v_{A} \delta \boldsymbol{v}, B_{0} \delta \boldsymbol{b}\right)$. In this case, the linear mode decomposition will be meaningful. After Fourier transforming the MHD data, the MHD state at each wave vector $\boldsymbol{k}$ is arranged in a column vector consisting of $\left(\rho_{\boldsymbol{k}}, \boldsymbol{v}_{\boldsymbol{k}}, \boldsymbol{b}_{\boldsymbol{k}}\right)$, where $\rho$ is the density, $v$ is the perturbed velocity field vector (normalized to the Alfvén speed), and $\boldsymbol{b}$ is the perturbed magnetic field vector (normalized to the mean field $\boldsymbol{B}_{0}$ ). Here, the mean density $\rho_{0}$ and mean magnetic field $\boldsymbol{B}_{0}$ have been subtracted, and only the fluctuating components are kept. The magnetic field components are not truly independent due to the divergence-free condition $\boldsymbol{k} \cdot \boldsymbol{b}_{\boldsymbol{k}}=0$. Therefore, only two components of the $\boldsymbol{b}$ field are kept to reduce the MHD state vector to six components. These two components are selected to be closest to the magnetic field vector of the Alfvén mode and the slow (or fast) mode.

The six eigenmode vectors are placed in a $6 \times 6$ matrix $A$, while the MHD state is a column vector $b$, solving for the linear amplitudes $x$ of the modes by solving the linear matrix equation $A x=b$. Once the Fourier amplitudes of the different modes are obtained in this manner, an inverse Fourier transform gives us data cubes in the physical space consisting entirely of single, specific MHD modes. The perpendicular propagation, $k_{z}=0$, is a special case where the Alfvén and slow modes become degenerate and nonpropagating. The parallel propagation case $\left(k_{\perp}=0\right)$ is also special, as here the Alfvén mode is degenerate with either the slow or fast mode, depending on whether $v_{A}<c_{s}$ or $v_{A}>c_{s}$.

Using this decomposition, the density, velocity, and magnetic fields obtained from MHD simulations are decomposed into three modes, i.e., $\boldsymbol{v}_{\boldsymbol{k}}=\boldsymbol{v}_{\boldsymbol{k}, A}+\boldsymbol{v}_{\boldsymbol{k}, S}+$ $\boldsymbol{v}_{\boldsymbol{k}, F}$ and $\boldsymbol{b}_{\boldsymbol{k}}=\boldsymbol{b}_{\boldsymbol{k}, A}+\boldsymbol{b}_{\boldsymbol{k}, S}+\boldsymbol{b}_{\boldsymbol{k}, F}$, where the subscripts $A$, $S$, and $F$ refer to the Alfvén, slow, and fast modes, 


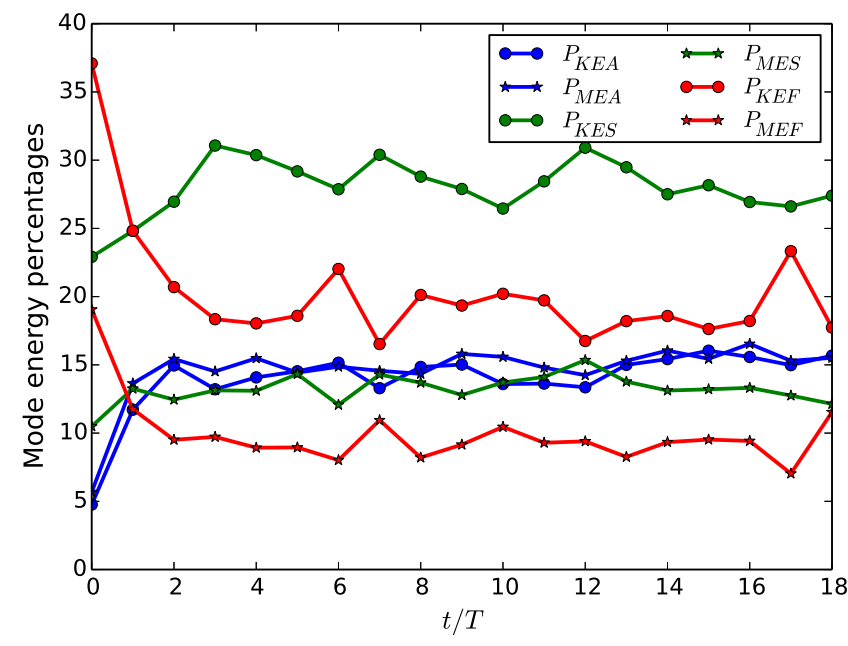

FIG. 1. Fraction of mode energies in the velocity and magnetic fields of the different MHD modes for the C1a simulation as a function of time.

respectively. To measure the relative presence of the different modes in the turbulence, the relative fraction of "energy" in that mode is calculated by taking

$$
\begin{aligned}
& P_{\mathrm{ME} m}=100 \% \times \frac{\sum_{k}\left|\boldsymbol{b}_{\boldsymbol{k}, m}\right|^{2}}{\sum_{m, \boldsymbol{k}}\left(\left|\boldsymbol{b}_{\boldsymbol{k}, m}\right|^{2}+\left|\boldsymbol{v}_{\boldsymbol{k}, m}\right|^{2}\right)}, \\
& P_{\mathrm{KE} m}=100 \% \times \frac{\sum_{\boldsymbol{k}}\left|\boldsymbol{v}_{\boldsymbol{k}, m}\right|^{2}}{\sum_{m, \boldsymbol{k}}\left(\left|\boldsymbol{b}_{\boldsymbol{k}, m}\right|^{2}+\left|\boldsymbol{v}_{\boldsymbol{k}, m}\right|^{2}\right)} .
\end{aligned}
$$

The variable $m$ represents the MHD modes, standing for $A$, $S$, or $F$. Here, $P_{\mathrm{ME} m}$ [Eq. (3)] stands for magnetic energy fractions and $P_{\mathrm{KE} m}$ [Eq. (4)] stands for kinetic energy fractions. The sum over $m$ in the denominator means summing over all three modes. The sum over the Fourier modes excludes the $k_{z}=0$ and $k_{\perp}=0$ modes, counting only nondegenerate modes. As an example, $P_{\text {MEA }}$ stands for the percentage of magnetic energy in Alfvén modes, while $P_{\mathrm{KEF}}$ estimates the fraction of fast-mode velocity fluctuations in the total mode mixture.

These mode energy fractions change as a function of time as shown in Fig. 1. It shows the energy fractions as a function of time in simulation C1a. This simulation is first run at a lower resolution of $128^{3}$ in order to reach a steady state in energy quickly. Then, the $512^{3}$ simulation is launched using a data cube from the $128^{3}$ simulation as the initial condition with trilinear interpolation. We see that it takes some time initially for the different mode fractions to attain a steady value. The Alfvén mode shows a very similar level of kinetic and magnetic fluctuations, as expected from its eigenmodes. The dominant contribution comes from the kinetic component of the slow modes, while its magnetic component is comparable to the Alfvén modes. The fast mode also shows a strong kinetic component and a weaker magnetic component.

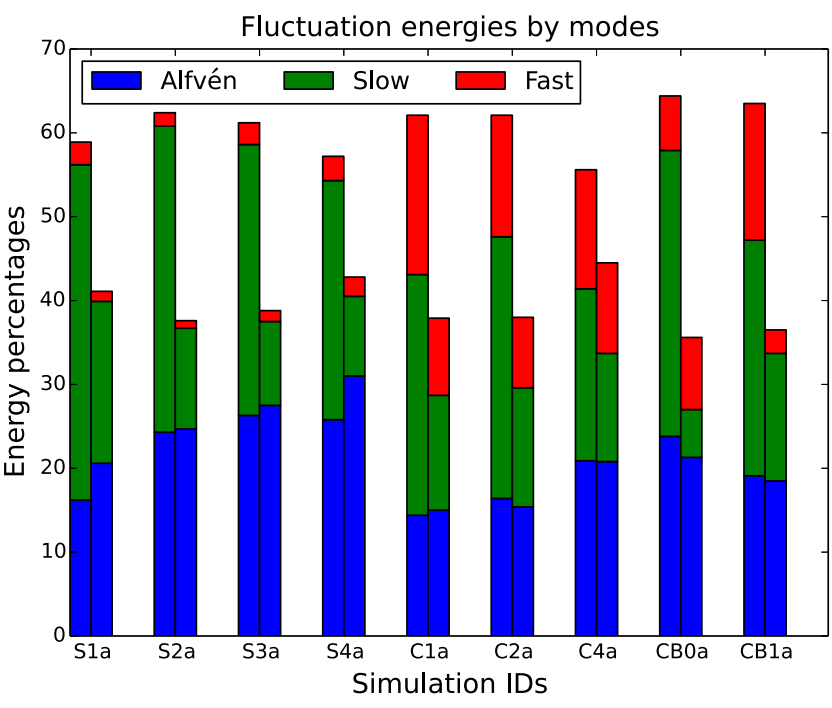

FIG. 2. The time-averaged fractions of mode energies in different modes in different simulations. Each simulation has two bars. The left one represents the velocity field showing the three mode percentages $\left(P_{\mathrm{KEA}}, P_{\mathrm{KES}}\right.$, and $P_{\mathrm{KEF}}$ in blue, green, and red, respectively). Similarly, the right bar is for the magnetic field showing $P_{\mathrm{MEA}}, P_{\mathrm{MES}}$, and $P_{\mathrm{MEF}}$ in their respective colors. Both the bars add up to $100 \%$. Compressive driving leads to a significantly larger fraction of the fast magnetosonic mode.

These mode energy fractions are averaged over the steady-state snapshots of each $512^{3}$ simulation and shown in Fig. 2. In the solenoidally driven simulations, the Alfvén and slow modes form the major fraction, with very little contribution from fast modes. In simulation S4a, the fast mode contributes only $5.1 \%$ to kinetic energy fluctuations and $5.4 \%$ to magnetic energy fluctuations. Going from S1 to $\mathrm{S} 4$ as the Alfvén Mach number increases, there is a slight increase in the fraction of Alfvén modes. The Alfvén modes have roughly equal energies in the velocity and magnetic fields, while the slow mode has a stronger component of velocity fields. A striking feature of Fig. 2 is that the fast mode has a significantly large proportion in the compressively driven simulations, which has not been observed before. In simulation $\mathrm{C} 1 \mathrm{a}$, the fast mode contributes $30.6 \%$ to kinetic energy fluctuations and $24.3 \%$ to magnetic energy fluctuations. In simulation $\mathrm{C} 4 \mathrm{a}$, the fast mode contributes $25.5 \%$ to kinetic energy fluctuations and $24.3 \%$ to magnetic energy fluctuations. On the other hand, comparing $\mathrm{C} 1 \mathrm{a}, \mathrm{C} 2 \mathrm{a}$, and $\mathrm{C} 4 \mathrm{a}$ shows that changing the $M_{A}$ does not affect the mode fractions significantly (except for a gradual increase in Alfvén-mode proportion). Comparing CB0a with CB1a shows that kinetic fluctuations of slow modes decrease while their magnetic fraction increases as the plasma $\beta$ increases, taking the slow-mode magnetic and kinetic fluctuations closer to equipartition. This case is understood from the fact that as $\beta \rightarrow \infty$, the slow-mode dispersion tends to the Alfvén-mode dispersion, which implies equipartition between kinetic and magnetic 
(a) Mag. Sla

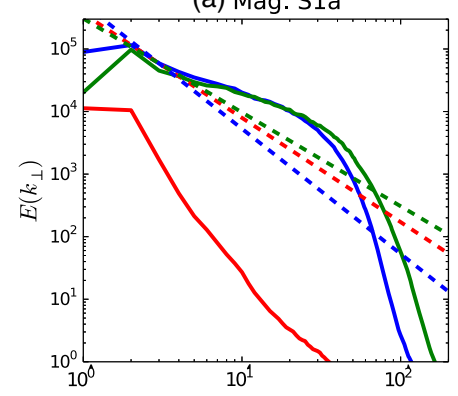

(e) Kin. Sla

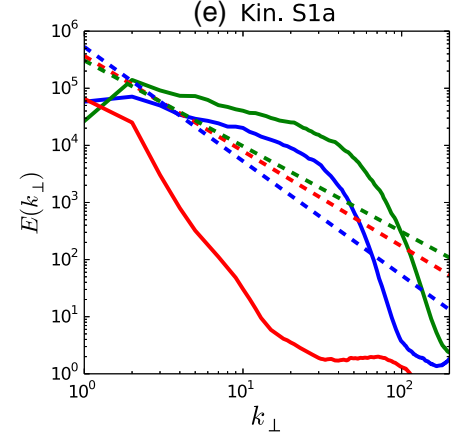

(b) Mag. S4a

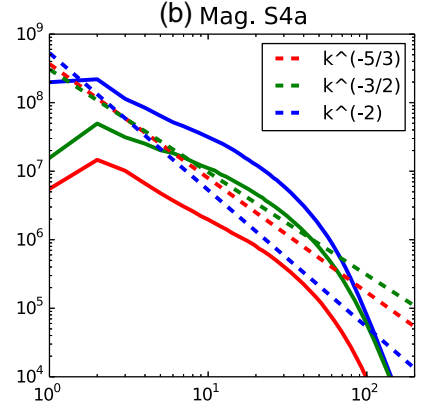

(f) Kin. S4a

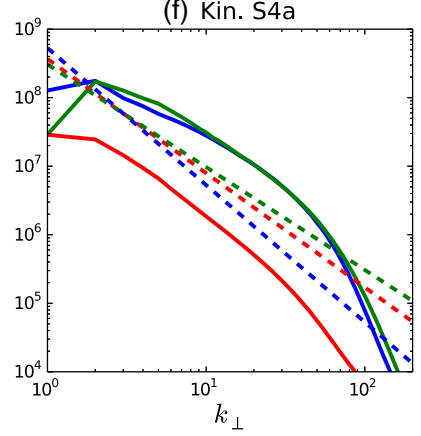

(c) Mag. Cla

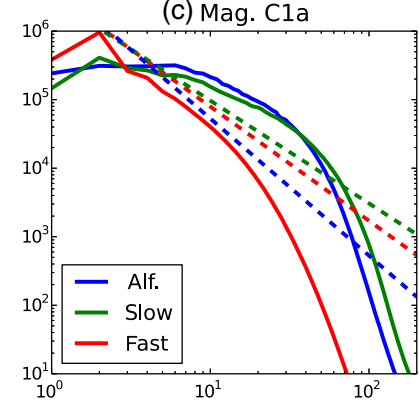

(g) Kin. Cla

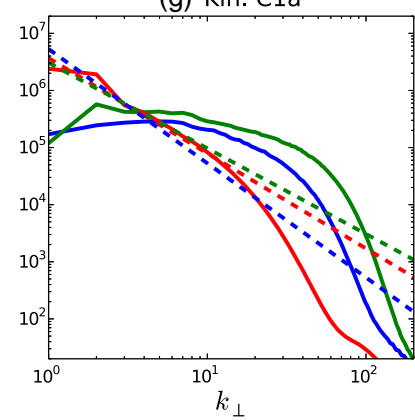

(d) Mag. C4a

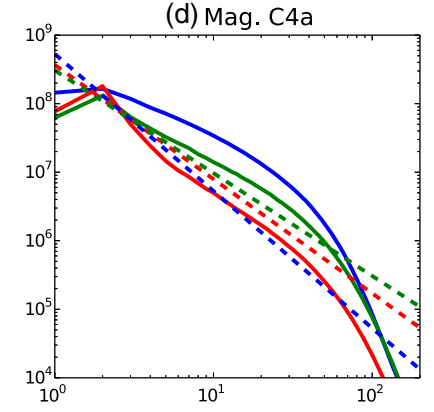

(h) Kin. C4a

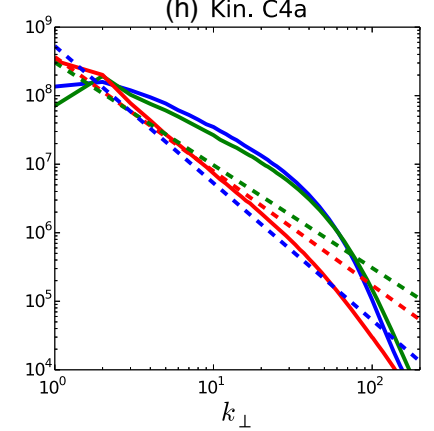

FIG. 3. Energy spectra of the different MHD modes showing a comparison between S1a, S4a, C1a, and C4a simulations. The top row is the spectrum of magnetic fields, and the bottom row shows the velocity field spectrum. Three reference slopes are also plotted giving $k_{\perp}^{-3 / 2}, k_{\perp}^{-5 / 3}$, and $k_{\perp}^{-2}$ slopes. The legend applies to all the subplots.

fluctuations. Also, as $\beta$ increases, the fraction of the fast mode increases in the kinetic fluctuations, while it decreases in the magnetic fluctuations. This result is expected from the fast-mode eigenvector [Eq. (A30) of Ref. [19] ], as in the high $\beta \rightarrow \infty$ limit, we have $\boldsymbol{v}_{\boldsymbol{k}, F} \propto \boldsymbol{k}$ and $\omega_{k, F} \sim k c_{s}$. This case gives $\left|\boldsymbol{b}_{k, F}\right| \sim\left|\boldsymbol{v}_{\boldsymbol{k}, F}\right| B_{0} / c_{s}$ and hence $\left|\boldsymbol{b}_{\boldsymbol{k}, F}\right| / B_{0} \ll\left|\boldsymbol{v}_{\boldsymbol{k}, F}\right| / v_{A}$.

If we consider only the velocity fluctuations, then in all the compressively driven simulations, the total fraction of slow and fast modes is larger than the fraction of the Alfvén mode. Since the Alfvén mode is incompressible while the fast and slow magnetosonic modes are compressible, compressive driving expectedly makes the compressible velocity components dominant. If we consider only the magnetic fluctuations, then in simulations $\mathrm{C} 1 \mathrm{a}, \mathrm{C} 2 \mathrm{a}$, and $\mathrm{C} 4 \mathrm{a}$, the slow-plus-fast fraction is larger than the Alfvén fraction. Therefore, even magnetic fluctuations are dominated by the compressible magnetosonic modes in compressively driven turbulence of plasma $\beta$ close to unity.

The mode fractions only give us a crude estimate of the strengths of various modes. We look at the perpendicular wave-number energy spectrum to get a sense of the wavenumber distribution of the mode energies. These spectra are shown in Fig. 3. The perpendicular direction is taken with respect to the $z$ direction, which is the mean field direction. The spectrum in the perpendicular $x-y$ plane is averaged over the angle $\theta$ between the $\boldsymbol{k}_{\perp}$ vector and the $x$ axis, $E\left(k_{\perp}\right)=\int d \theta k_{\perp} E\left(k_{\perp}, \theta\right)$. This spectrum is very similar to the $1 \mathrm{D}$ wave-number spectrum averaged over all three directions, $E(k)$. The $k_{z}=0$ wave number is removed from the data cube when calculating this spectrum in order to be consistent with Fig. 2. The energy spectra of the Alfvén and slow modes show very similar behavior across all the cases. The solenoidally driven low- $M_{A}$ case S1a is a case where the Alfvén cascade is weak, as we will see later. The fast mode in this case is very weak energetically, compared to the slow and Alfvén modes, with a very steep spectrum. In the solenoidally driven trans-Alfvénic case S4a, the Alfvén and slow modes show a spectrum close to $k_{\perp}^{-3 / 2}$, which is indicative of strong turbulence. The magnetic field spectrum of fast modes is close to $k_{\perp}^{-3 / 2}$, while the velocity field spectrum is between $k_{\perp}^{-5 / 3}$ and $k_{\perp}^{-2}$. This case is similar to earlier results of fast-mode spectra [19].

In the compressively driven cases of $\mathrm{C} 1 \mathrm{a}$ and $\mathrm{C} 4 \mathrm{a}$, we see that the fast-mode energy level increases compared to the solenoidally driven cases at large scales close to the driving scales. In $\mathrm{C} 4 \mathrm{a}$, the Alfvén and slow modes still show very similar spectra, close to $k_{\perp}^{-3 / 2}$. The fast-mode velocity field in both $\mathrm{C} 1 \mathrm{a}$ and $\mathrm{C} 4 \mathrm{a}$ shows a spectrum of $k_{\perp}^{-2}$, while the magnetic field spectrum is between $k_{\perp}^{-5 / 3}$ and $k_{\perp}^{-2}$. This spectrum is steeper than the $k^{-3 / 2}$ spectrum claimed in Ref. [19]. Sharp jumps in data can lead to a steeper power spectrum [38] but might also limit the applicability of the mode decomposition. We try to identify regions of these sharp gradients in a fast-mode data cube of simulation $\mathrm{C} 4 \mathrm{a}$ by finding cells that have velocity jumps in neighboring cells above a threshold. Regions with more than a $30 \%$ 
jump in the velocity occupy $10 \%$ of the total volume (more than $100 \%$ jump regions occupy only $0.8 \%$ volume). These sharp gradients are weak and occupy a very small volume; therefore, we expect that the mode decomposition would still be valid for such data. To test this case further, we perform a test simulation with a superposition of three fast modes with mutually orthogonal wave vectors that do not interact via radial three-wave interactions. These modes steepen into shocks, but the mode decomposition of the data still reveals the dominance of fast modes, as is expected. Therefore, we can rely on mode decomposition even in this scenario. As the slope of the fast-mode spectrum is steeper, even though it is dominant close to the driving scale, its energy component drops off compared to the Alfvén and slow modes at smaller scales.

The spectrum of Alfvén and slow modes in simulations S1a and C1a shown in Fig. 3 is shallower than $k_{\perp}^{-3 / 2}$ and closer to $k_{\perp}^{-1}$. These simulations were run for $T=16$, which is close to an order unity nonlinear time. We also ran the simulations for a longer time up to $T=32$ and verified that the spectra converged with time. As the forcing injects energy in the velocity field isotropically in a spherical shell of wave vectors with $1 \leq k \leq 3$, this could be a case of weak turbulence driven hydrodynamically at both $k_{z} \neq 0$ and $k_{z}=0$ as considered in Ref. [39]. The Alfvén modes appear with a similar spectrum in the $\mathrm{S} 1$ simulation. The spectrum of $k_{z} \neq 0$ modes is $k_{\perp}^{-1}$ as predicted [see Fig. 4(a)]. The OU forcing plays an important role in producing this behavior. We implemented and tried the delta-correlated-in-time forcing often used in turbulence simulations like in Refs. [19,40-42] and produced a turbulent date cube with $M_{A}=0.24$. We compare the spectrum produced by this simulation with the OU forced simulation S1a in Fig. 4. Theoretically, a large range of
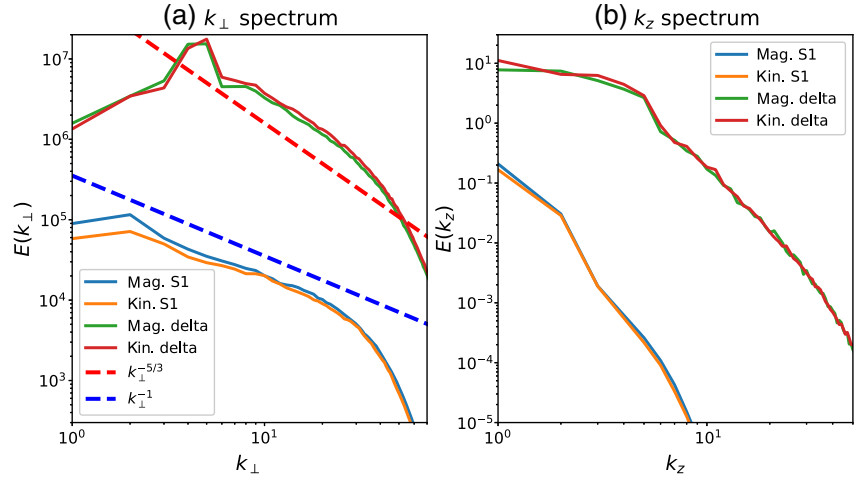

FIG. 4. (a) The $k_{\perp}$ spectrum excluding the $k_{z}=0$ mode. A delta-correlated-in-time forcing is used to produce a data cube with $M_{A}=0.24$ (labeled as "delta"), and it is compared with the OU-process forced S1 simulation. The delta-correlated forcing produces a spectrum close to $k_{\perp}^{-5 / 3}$, while the $\mathrm{S} 1$ simulation has a spectrum $k_{\perp}^{-1}$. (b) Comparison of the $k_{z}$ spectrum. The OU forcing produces a very weak energy cascade to higher $k_{z}$ modes, while the delta-correlated forcing produces a stronger cascade. weak turbulence is expected from $k_{\text {inj }}$ to $k_{\text {inj }} / M_{A}^{2}$ [43]. In the OU forced simulation, the $k_{z}$ spectrum is very steep, indicating weak turbulence, while the $k_{\perp}$ spectrum is $k_{\perp}^{-1}$. On the other hand, the delta-correlated forcing produces a $k_{\perp}^{-5 / 3}$ spectrum, which is more representative of strong turbulence along with a significant energy cascade to higher $k_{z}$ modes. The energy spectra of $\mathrm{OU}$ forced simulations in Fig. 4 are also lower than the delta-correlated spectra since, for the OU simulations, more energy is concentrated in the $k_{z}=0$ modes. We think that the deltacorrelated-in-time forcing produces faster dynamics, whereas the OU forcing gives a slower evolution of the force allowing a weak cascade to develop.

We can also see signatures of different modes in the frequency spectra. For this case, one-dimensional slices along the $x$ (perpendicular to the mean field) and $z$ (parallel to the mean field) axes are taken. These slices are output at a high frequency at a time interval of $\Delta t=0.002 \tau_{A}$. Then, performing a 2D Fourier transform along the $x(z)$ and time axes gives us the power distribution in $k_{\perp}\left(k_{z}\right)-\omega$ space. Figure 5 shows this power spectrum in the $k_{z}-\omega$ space. The white dashed line follows the $\omega= \pm k_{z} v_{A}$ line, which is the dispersion relation of Alfvén waves. The green dashed line traces the relation $\omega=(1+\sqrt{2}) \sqrt{2} k_{z} v_{A}$. This is the relation for a fast mode where $c_{s}=v_{A}$ (approximately true for these simulations) and $k_{\perp}=k_{z}$. Figure 5(a) shows the frequency spectrum from the velocity fluctuations in simulation S2a, in which the Alfvén- and slowmode contributions dominate significantly over fast modes as seen in Fig. 2. We see that the power is concentrated close to the Alfvénic dispersion. Figure 5(b) shows the frequency spectrum for the velocity field in simulation C2a, which also shows a branch of power concentrated at higher frequencies $\omega$ that are close to the fast-mode dispersion. The fast mode is also a significant proportion of the mode mixture in the C2a simulation (Fig. 2), and this is reflected in the frequency characteristics. In the next section, we
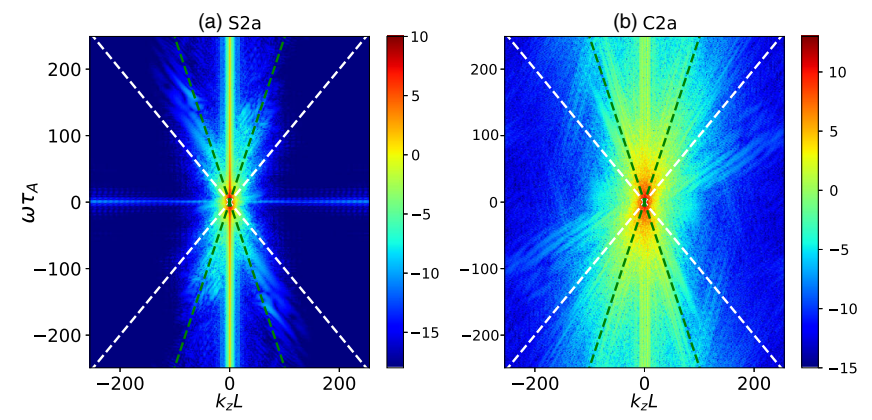

FIG. 5. Frequency spectra showing power density in $\omega$ versus $k_{z}$. The color represents the logarithm of power. The left plot is from the velocity field of simulation $\mathrm{S} 2 \mathrm{a}$, while the right plot is for simulation $\mathrm{C} 2 \mathrm{a}$, again using the velocity field. The white dashed line is a reference Alfvén-mode dispersion, while the dashed green line is an example of fast-mode dispersion. 
focus on the anisotropy characteristics of the Alfvén and slow modes.

\section{ALFVÉN AND SLOW MODES}

We are interested in the nature of the cascade of the different MHD modes, especially in its anisotropy. Thus, we analyze the energy spectrum in the $k_{z}-k_{\perp}$ space. Here, the parallel direction is along the mean field, i.e., along the $z$ direction. The 2D spectrum is defined as $E\left(k_{z}, k_{\perp}\right)=\int k_{\perp} E\left(k_{\perp}, \theta, k_{z}\right) d \theta$, where $k_{z}$ is parallel to the mean magnetic field, $k_{\perp}=\sqrt{k_{x}^{2}+k_{y}^{2}}$, and $\theta$ is the angle between $\boldsymbol{k}_{\perp}$ and the $x$ axis. Figure 6 shows these spectra for the velocity field of the Alfvén modes in simulations S1a-S4a with increasing $M_{A}$. We see that for S1a, the energy is distributed along the $k_{\perp}$ axis close to $k_{z}=0$. There is very little cascade along the parallel direction in the sense that as energy spreads to higher $k_{\perp}$, there is no spread to higher $k_{z}$. The situation is similar for the S2a simulation. As the $M_{A}$ increases, the cascade slowly spreads in the parallel direction, slightly in the S3a simulation and more prominently in the S4a simulation. This result is an indication of the turbulence transitioning from weak to strong as $M_{A}$ increases.

We further analyze the anisotropy of the Alfvén modes by using structure functions. The anisotropic structure function is defined as
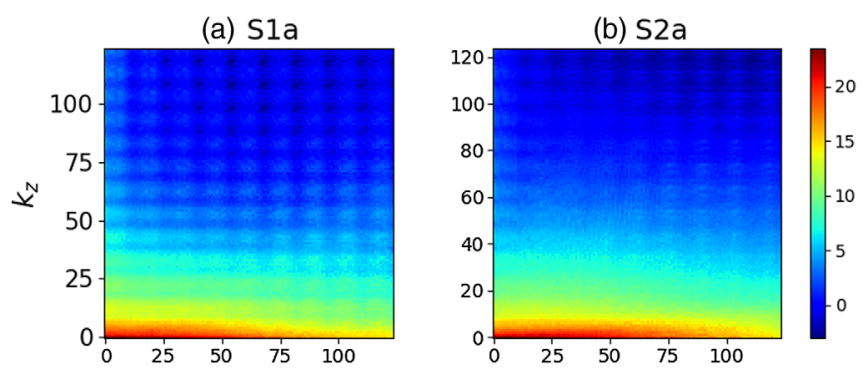

(c) $\mathrm{S3a}$

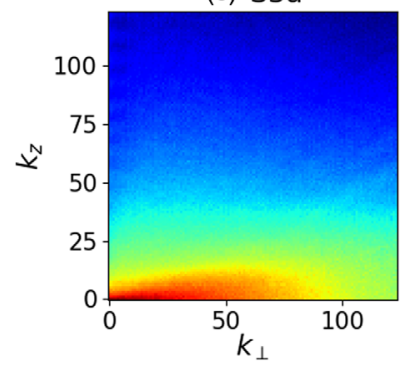

(d) $\mathrm{S} 4 \mathrm{a}$

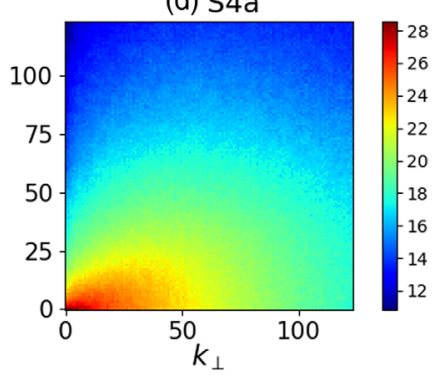

FIG. 6. The $k_{z}-k_{\perp}$ wave-number spectrum for the velocity field of Alfvén modes with increasing Mach number. The color indicates the logarithm of the spectrum power. S1a is the lowest Mach number of 0.24 , going up to $\mathrm{S} 4 \mathrm{a}$, which has the highest Mach number of 0.99 . The power spreads more in the parallel direction as the Alfvén Mach number increases.

$$
\begin{aligned}
S F_{2}\left(l_{\|}, l_{\perp}\right)= & \langle| \boldsymbol{b}\left(\boldsymbol{r}-\left(l_{\|} / 2\right) \hat{\boldsymbol{b}}-\left(l_{\perp} / 2\right) \hat{\boldsymbol{b}}_{\perp}\right) \\
& \left.-\left.\boldsymbol{b}\left(\boldsymbol{r}+\left(l_{\|} / 2\right) \hat{\boldsymbol{b}}+\left(l_{\perp} / 2\right) \hat{\boldsymbol{b}}_{\perp}\right)\right|^{2}\right\rangle_{\boldsymbol{r}} .
\end{aligned}
$$

This function involves an ensemble average over a number of pairs of points, which are separated by distance $l_{\|}$in the magnetic field parallel direction $(\hat{\boldsymbol{b}})$ and distance $l_{\perp}$ in the field-perpendicular direction $\left(\hat{\boldsymbol{b}}_{\perp}\right)$. The magnetic field direction $\hat{\boldsymbol{b}}$ is the local mean magnetic field. To obtain the local mean magnetic field, for each parallel and perpendicular distance pair $\left(l_{\|}, l_{\perp}\right)$, a distance $l=\sqrt{l_{\|}^{2}+l_{\perp}^{2}}$ is first calculated. Then, a random point is selected in the data cube, and a sphere is taken around this point with a diameter $l$. The local mean magnetic field direction is calculated by taking an average of a few $(\geq 5)$ random points located in this sphere. We have verified that the results do not change when taking more points, which gives us the local mean field direction $\hat{\boldsymbol{b}}$, and a random unit vector perpendicular to $\hat{\boldsymbol{b}}$ is also constructed, $\hat{\boldsymbol{b}}_{\perp}$. Now we select two points on this sphere that are separated by $l_{\|} \hat{\boldsymbol{b}}+l_{\perp} \hat{\boldsymbol{b}}_{\perp}$. An ensemble average over thousands of such pairs gives us $S F_{2}\left(l_{\|}, l_{\perp}\right)$.

The isocontours of the structure function are plotted in Fig. 7. A second-order smoothing is applied a few times on the structure function to make the contours smoother, without changing their behavior. These are derived from the magnetic and velocity fields of the decomposed Alfvén mode in the four different simulations S1b, S4b, C2a, and $\mathrm{CB} 1 \mathrm{a}$, averaged over several time snapshots. The driving occurs up to a length scale of 0.33 units in terms of box length, so we only focus on $l_{\|}$and $l_{\perp}$ up to 0.2 . The anisotropy of the Alfvén modes is clearly visible across the different simulations and is quite similar. The parallel length scales are larger than the perpendicular length scales. Moreover, the ratio $l_{\|} / l_{\perp}$ changes with $l_{\|}$, with this anisotropy increasing as we proceed to smaller scales, making this a scale-dependent anisotropy. Comparing S4b and C2a simulations shows that the anisotropy of Alfvén modes is not affected by the type of driving. Changing the plasma $\beta$ in simulation CB1a does not seem to change the anisotropy either. Simulation S1b shows a structure function of the velocity field that is highly elongated along the $l_{\|}$direction. Similar behavior is seen for the larger $l_{\perp}$ values in $\mathrm{C} 2 \mathrm{a}$ and $\mathrm{CB} 1 \mathrm{a}$ velocity field structure functions, which are low $M_{A}$ simulations. We observe that at low $M_{A}$, the velocity field is highly correlated along the magnetic field direction, giving rise to an almost $l_{\|}$-invariant structure function. This case is indicative of the weak nature of the cascade at low $M_{A}$. As $M_{A}$ increases, smaller-scale structure develops in the parallel direction. The trans-Alfvénic simulation S4b shows a structure function that has smaller $l_{\|}$scales. We need to look further at the variation of $l_{\|}$with $l_{\perp}$ to get a quantitative understanding of the anisotropy of Alfvén modes. 


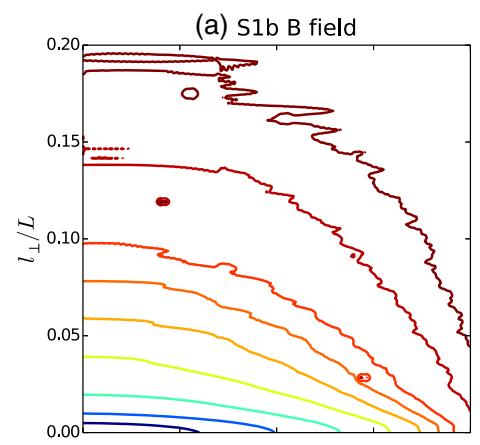

(e) S1b v field

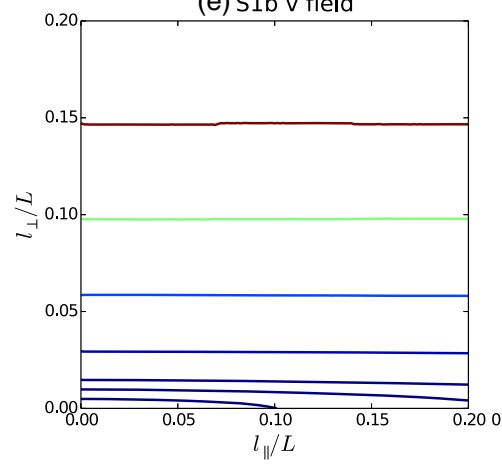

(c) C2a B field

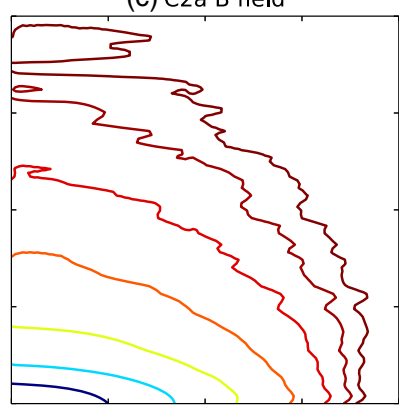

(g) C2a v field

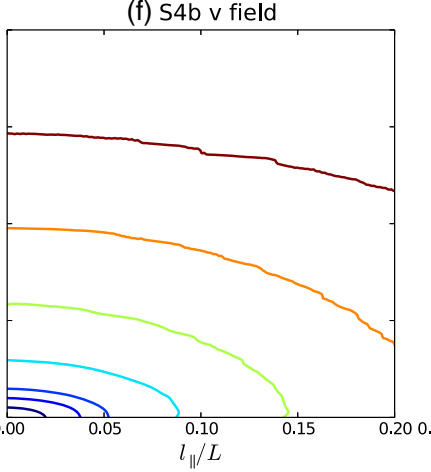

(b) S4b B field

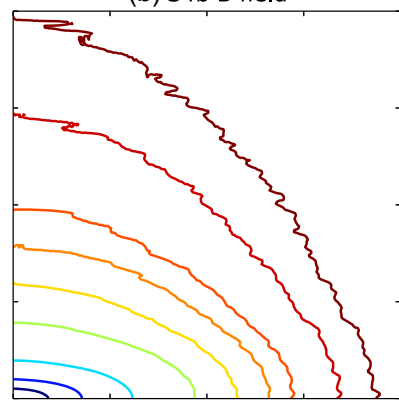

(f) S4b v field

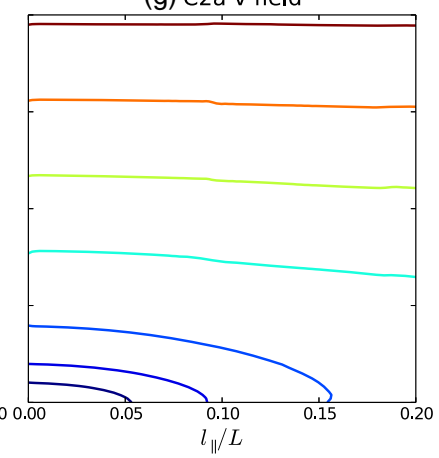

(d) CBla B field

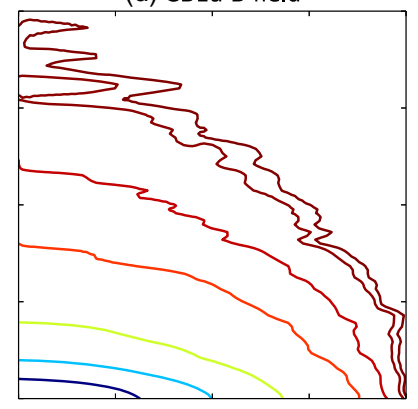

(h) CBla v field

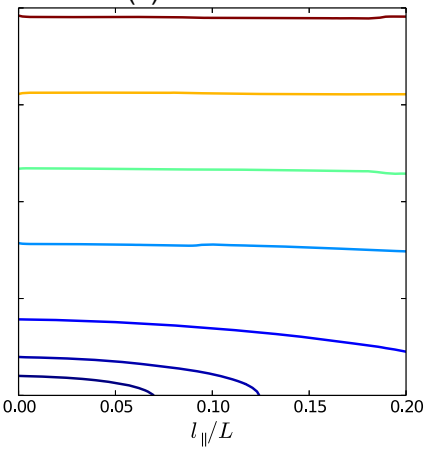

FIG. 7. Isocontours of the structure function for Alfvén modes from their magnetic (top panels) and velocity fields (bottom panels) for the four simulations S1b, S4b, C2a, and CB1a. The units of $l_{\|}$and $l_{\perp}$ are in terms of the simulation box size. We see the scale-dependent anisotropy for the Alfvén modes, which are longer in the parallel direction compared to the perpendicular direction.

We also analyze the 2D structure functions of the slow modes. They show a behavior similar to the Alfvén modes with an anisotropy showing $l_{\|}>l_{\perp}$ at all scales.

From the 2D structure functions, we can extract the relation of $l_{\|}$versus $l_{\perp}$ by taking the cuts of the isocontours of a constant structure function at the $l_{\|}$and $l_{\perp}$ axis. For this process, the structure function along the two axes $\left(l_{\|}=0\right.$ and $l_{\perp}=0$ ) is calculated with very high statistics such that a smooth interpolation can be used to obtain a relation between the $l_{\|}$and $l_{\perp}$. This measure gives us a better picture of the anisotropy scaling. Figure 8 shows this measure derived from the magnetic fields of the Alfvén modes. We see that the anisotropy depends on the $M_{A}$ for the Alfvén modes. For simulation $\mathrm{S} 1 \mathrm{~b}$, the $l_{\|}$is almost constant (very weakly changing) at large $l_{\perp}$, implying that eddies form at smaller perpendicular length scales but maintain the same parallel length scales, which is an indication of weak turbulence. For simulation S3b, a similar behavior is also observed at large $l_{\perp}$, but the $l_{\|}$ starts decreasing as $l_{\perp}$ gets smaller. As the Mach number increases in simulation $\mathrm{S} 4 \mathrm{~b}$, the $l_{\|}$scales close to the Goldreich-Sridhar scaling of $l_{\|} \sim l_{\perp}^{2 / 3}$. The $\mathrm{C} 4 \mathrm{~b}$ simulation shows a power law with the Goldreich-Sridhar scaling for a significant range of $0.02 \lesssim l_{\perp} \lesssim 0.1$. Comparing S1b and $\mathrm{C} 1 \mathrm{~b}$ shows not much difference between solenoidal and compressive driving. Comparing $\mathrm{CB} 0 \mathrm{a}$ with $\mathrm{CB} 1 \mathrm{a}$ shows that the plasma $\beta$ does not have a significant effect on the anisotropy. This shows that at low $M_{A}$ there is a large range of scales in $l_{\perp}$ where $l_{\|}$remains unchanging with $l_{\perp}$, indicating no cascade in parallel direction. As $M_{A}$ increases, this range decreases and we start seeing a transition to the Goldreich-Sridhar scaling.

Figure 9 shows the variation of $l_{\|}$, with $l_{\perp}$ derived from the magnetic fields of the slow modes. It shows a behavior similar to the Alfvén modes. For simulations S1b, S3b,

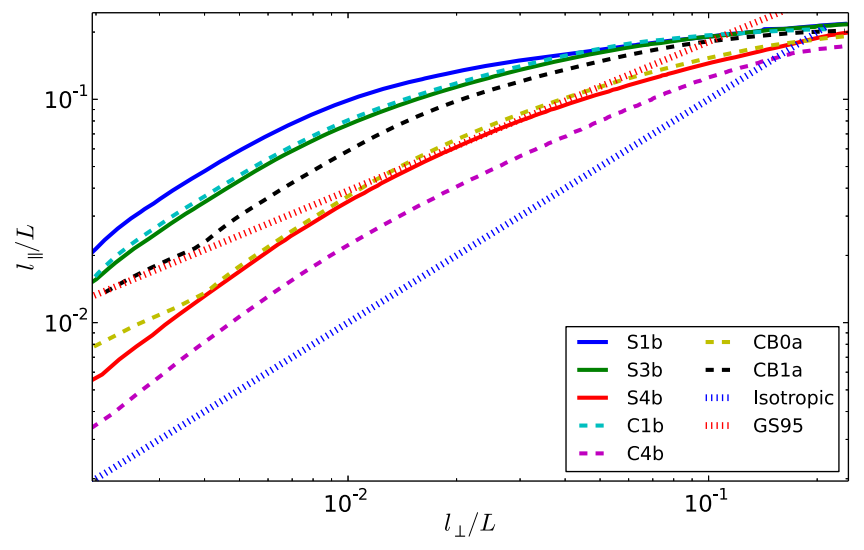

FIG. 8. The variation of $l_{\|}$versus $l_{\perp}$ for the Alfvén-mode magnetic field for the different simulations. The GoldreichSridhar scaling is $l_{\|} \sim l_{\perp}^{2 / 3}$, shown by the red dotted line (GS). Isotropic behavior $l_{\|}=l_{\perp}$ is shown by the blue dotted line. 


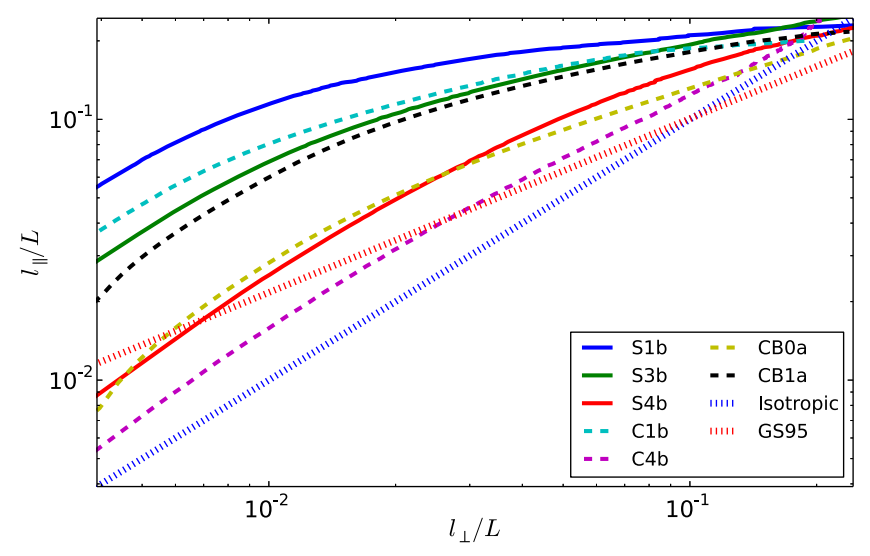

FIG. 9. The variation of $l_{\|}$versus $l_{\perp}$ for the slow-mode magnetic field for the different simulations. The GoldreichSridhar scaling is $l_{\|} \sim l_{\perp}^{2 / 3}$, shown by the red dotted line (GS). Isotropic behavior $l_{\|}=l_{\perp}$ is shown by the blue dotted line.

$\mathrm{C} 1 \mathrm{~b}$, and $\mathrm{CB} 1 \mathrm{a}$, the $l_{\|}$drops very slowly with decreasing $l_{\perp}$, which is similar to the weak nature of Alfvén modes in Fig. 8. Simulations CB0a, C4b, and S4b show a behavior close to Goldreich-Sridhar scaling. There is some isotropic scaling also seen in simulation C4b. This scaling could be due to coupling with the fast mode, which is strong in this case and shows isotropic scaling as we will see later.

The Alfvén cascade is expected to transition from weak to strong at a transition scale $\lambda_{\mathrm{CB}}$. The weak turbulence spectrum of Alfvén modes is $E\left(k_{\perp}\right) \sim\left(\epsilon / \tau_{A}\right)^{1 / 2} k_{\perp}^{-2}$ $[39,44]$, where $\epsilon$ is the energy injection rate. Then, $\lambda_{\mathrm{CB}}$ is the scale where the linear interaction time, $\tau_{A}=L / v_{A}$, balances the nonlinear interaction time, $\tau_{\mathrm{nl}}=\lambda_{\mathrm{CB}} / \delta v_{\lambda_{\mathrm{CB}}}$. From the weak turbulence spectrum, the velocity strength goes as $\delta v_{l_{\perp}} \sim l_{\perp}^{1 / 2}$. If we assume a velocity field of strength $v_{A} M_{A}$ at injection scale $L$, then $\delta v_{\lambda_{\mathrm{CB}}} \sim M_{A} v_{A}\left(\lambda_{\mathrm{CB}} / L\right)^{1 / 2}$. Balancing the linear and nonlinear interaction times gives $\lambda_{\mathrm{CB}} \sim L M_{A}^{2}[43,45]$. Thus, when $M_{A} \lesssim 1$, the weak regime of Alfvénic turbulence should exist in the range of scales $\left[L M_{A}^{2}, L\right]$, while at smaller scales, it should be in the strong regime.

We try to estimate this transition scale by making use of the structure function anisotropy. As seen in Fig. 8, the $l_{\|}$is expected to be invariant as a function of $l_{\perp}$ in the weak turbulence regime (at large $l_{\perp}$ ) and tend towards the Goldreich-Sridhar slope of $l_{\|} \sim l_{\perp}^{2 / 3}$ in the strong regime. Therefore, we fit a power law of the form $l_{\|}=C l_{\perp}^{\alpha}$ at each $l_{\perp}$ in a window of $l_{\perp}-\Delta$ to $l_{\perp}+\Delta$ around it. We take $\Delta=5$ grid points, and the scaling exponent $\alpha$ is calculated at each $l_{\perp}$. As expected at large scales close to the driving scale, the exponent is very close to 0 , and it increases as $l_{\perp}$ decreases. It crosses the Goldreich-Sridhar value of $2 / 3$ for the first time at some $l_{\perp}$ value, which can be taken as the transition scale $\lambda_{\mathrm{CB}}$. Thus, starting from large $l_{\perp}$, as we go down to smaller $l_{\perp}$, we define the transition scale $\lambda_{\mathrm{CB}}$ as the

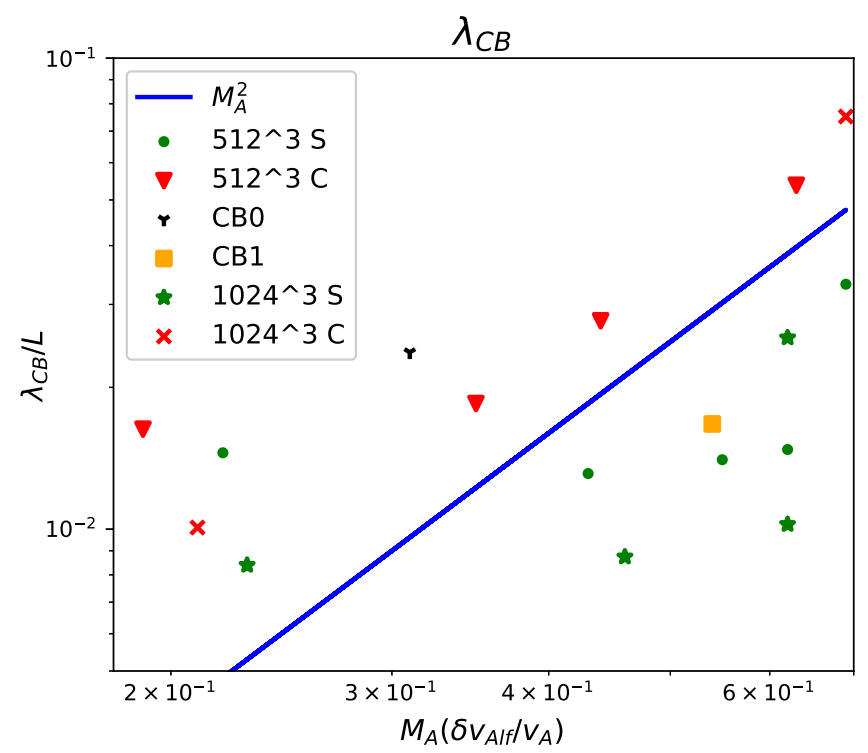

FIG. 10. The variation of the estimated transition scale of weak to strong turbulence $\lambda_{\mathrm{CB}}$ with the Alfvénic Mach number $M_{A}$. The different markers are the results from the different simulations, where $\mathrm{S}$ stands for solenoidal and $\mathrm{C}$ for compressive runs. The Alfvénic Mach number is calculated from the decomposed Alfvén data. The blue line shows the $M_{A}^{2}$ reference line.

$l_{\perp}$ at which $\alpha$ goes over a threshold value of $2 / 3$ for the first time. Thus, $\lambda_{\mathrm{CB}}$ is identified for the Alfvén mode in all the different simulations. The variation of this transition scale as a function of $M_{A}$ for each of these simulations is shown in Fig. 10. As the weak-to-strong transition is expected for the Alfvén cascade, we take the $M_{A}$ of the decomposed Alfvén mode instead of that of the total data. It is broadly indicative of the $M_{A}^{2}$ dependence as expected from theory. At low $M_{A}$, the $\lambda_{\mathrm{CB}}$ is already on scales close to the dissipative scales. Therefore, we see a plateau at low $M_{A}$. However, the trend is clearer from the simulations with $M_{A} \gtrsim 0.4$. Although there are only a few points with significant scatter and there also appears to be some systematic variation of the transition scale with plasma $\beta$ and type of forcing, power-law fits to these points are close to $M_{A}^{2}$ scaling. This verification is important for the existence of the weak regime of Alfvén turbulence. This feature needs to be taken into account when developing models of interstellar medium turbulence for cosmic-ray scattering. Next, we focus on the properties of the fast mode.

\section{FAST MODES}

In Fig. 3, the fast modes showed a different spectrum compared to the Alfvén and slow modes. Figure 11 shows the fast-mode wave-number spectrum as a function of $k_{\perp}$ and $k_{z}$. Contrasting it with Fig. 6, we see a different nature of the cascade here. For the Alfvén mode, the cascade is clearly anisotropic, with the energy cascade taking place 
(a) Sla

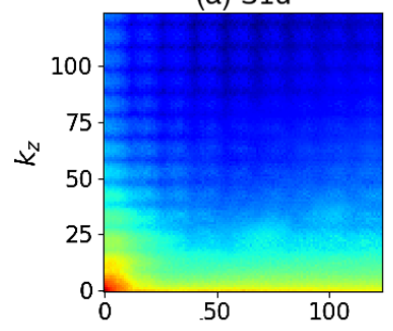

(c) $\mathrm{Cla}$

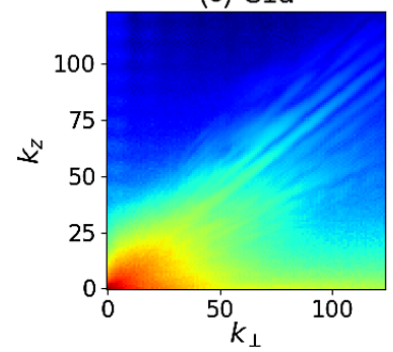

(b) $54 a$

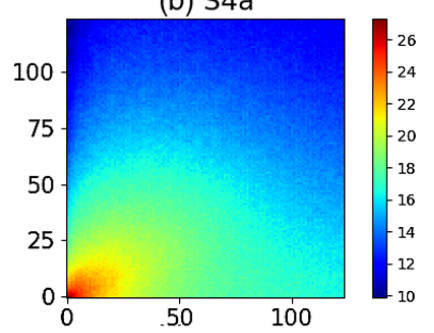

(d) $\mathrm{C} 4 \mathrm{a}$

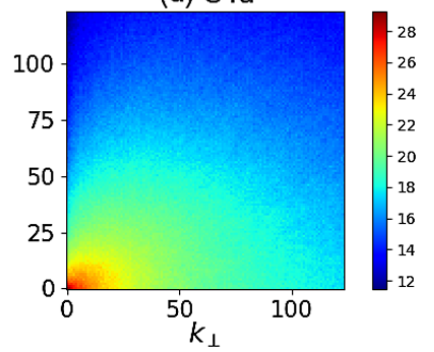

FIG. 11. The $k_{z}-k_{\perp}$ spectrum of the velocity field of fast modes for the four simulations S1a, S4a, C1a, and C4a.

mostly in the direction of larger $k_{\perp}$. However, the spread of energy for the fast mode appears very close to isotropic as there is almost uniform distribution of power in the parallel and perpendicular wave numbers. In the low- $M_{A}$ case of S1a where the fast mode has a tiny fraction, the energy distribution seems to be isotropic at low $k$, with a small

anisotropic cascade along the $k_{\perp}$ direction for higher $k_{\perp}$. However, for the other three simulations S4a, C1a, and $\mathrm{C} 4 \mathrm{a}$, the cascade extends radially. Another aspect is that the cascade for the Alfvén mode changes with $M_{A}$ in Fig. 6, with almost no cascade in the parallel direction for the S1a case due to the weak nature of turbulence. Here, in both $\mathrm{C} 1 \mathrm{a}$ and $\mathrm{C} 4 \mathrm{a}$ simulations, the fast mode shows an isotropic cascade. The isotropic nature of the fast-mode cascade is similar, even with solenoidal and compressive driving. This figure shows that the isotropic nature of the fast-mode cascade is a robust feature.

The 2D structure function isocontours of the velocity and magnetic fields of fast modes are shown in Fig. 12. These contours also show the isotropic nature for the various simulations. For the $\mathrm{C} 2 \mathrm{a}$ and $\mathrm{C} 4 \mathrm{~b}$ simulations, we can see the isotropic contours extending almost up to $l_{\perp} / L \sim 0.2$, which is close to the driving scale of 0.3. Also, in the $1024^{3}$ simulations, this isotropy extends well up to scales smaller than $l_{\perp} / L \sim 0.05$. From these results, we can say that the isotropy does indeed extend throughout the inertial range. The magnetic field contours in $\mathrm{C} 2 \mathrm{a}$ show a slight anisotropy at large $l_{\|}$. However, the contours from the velocity field are highly isotropic. From Fig. 2, we know that in simulation $\mathrm{C} 2 \mathrm{a}$, the fast-mode fraction is larger in the velocity field than in the magnetic field, so the velocity field shows a clearer isotropy. Cases CB0a and S2b show a little anisotropy, similar to Alfvén modes. These are cases where the fast-mode fraction is smaller (Fig. 2) compared (a) S2b B field

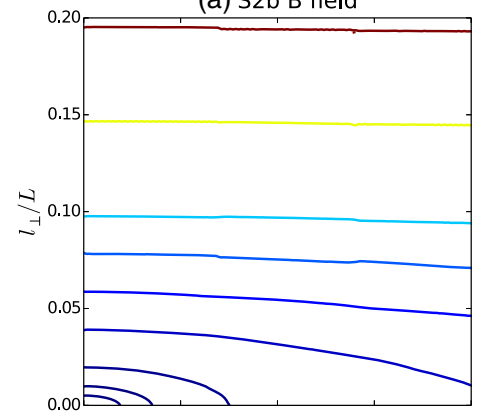

(e) S2b v field

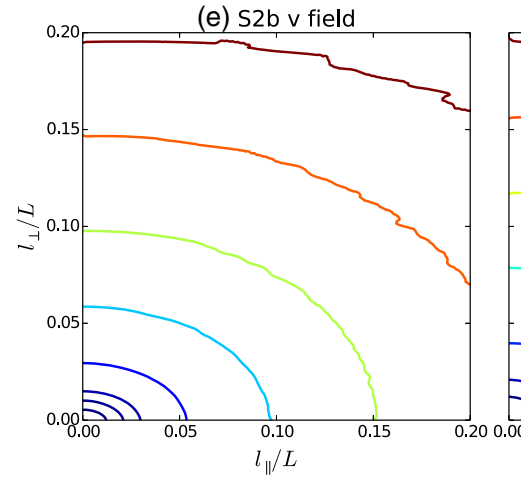

(b) C2a B field

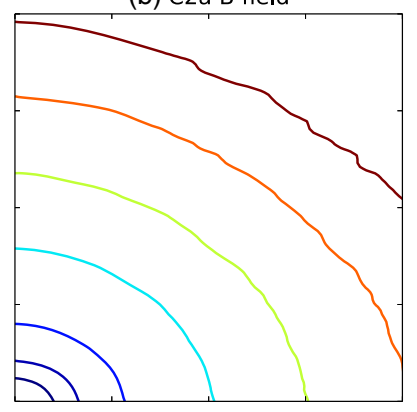

(f) C2a v field

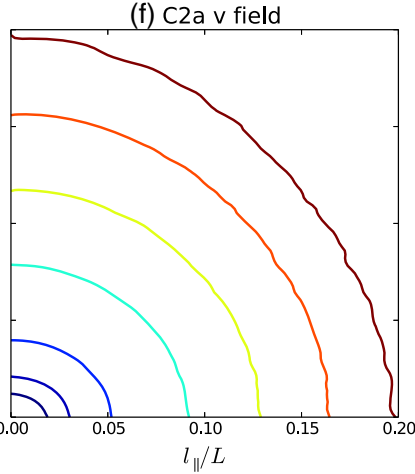

(c) C4b B field

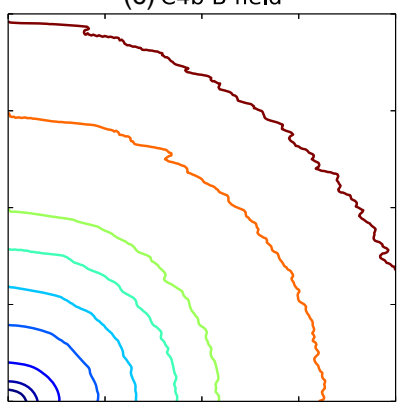

(g) C4b v field

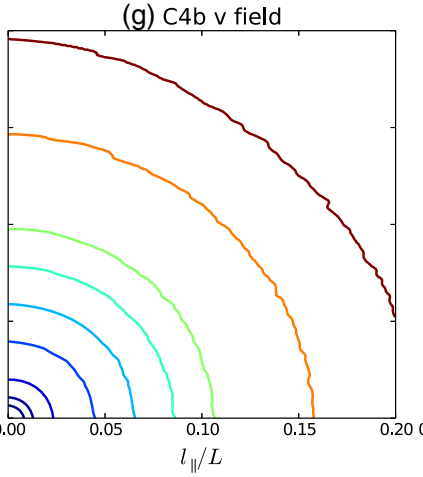

(d) CBOa B field

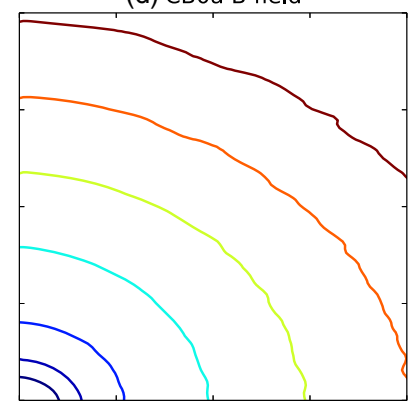

(h) CBOa v field

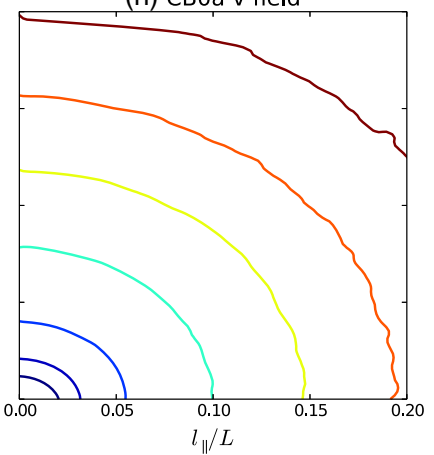

FIG. 12. The 2D structure functions for the fast mode for magnetic fields (top panels) and velocity fields (bottom panels) for the simulations S2b(a,e), C2a(b,f), C4b(c,g), and CB0a(d,h). They seem to be isotropic for the cases C2a, C4b, and CB0a, while for S2b, there seems to be some anisotropy. 

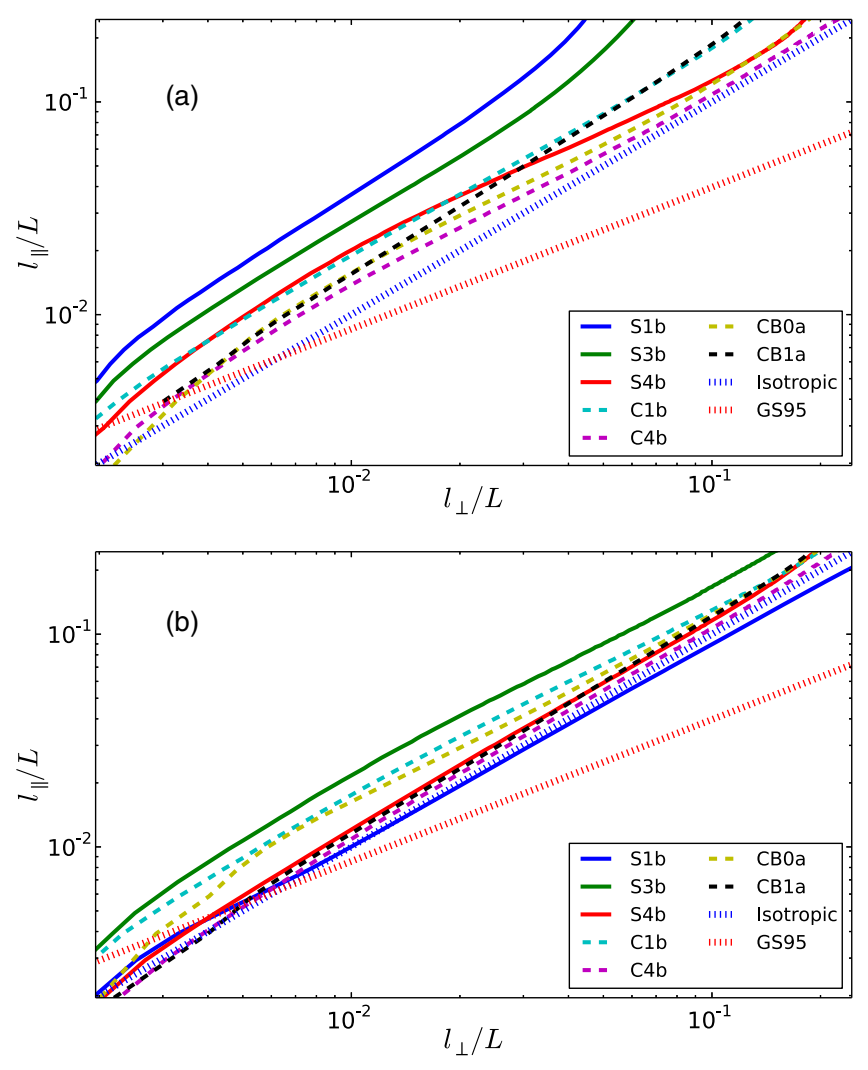

FIG. 13. The variation of $l_{\|}$versus $l_{\perp}$ for the fast modes for the (a) magnetic field and (b) velocity field. The blue dotted line shows isotropic behavior, while the red dotted line shows the Goldreich-Sridhar scaling.

to other cases, which indicates that the properties of a mode might be influenced by how strong it is compared to the other modes. If it is weak, then its properties might get influenced by the dominant mode properties. This observation points to the cascades of these modes not being entirely independent of each other, with some interaction between them.

We calculate the variation of $l_{\|}$with $l_{\perp}$ for the fast modes now. Figure 13(a) shows this calculation for the fast-mode magnetic field, while Fig. 13(b) is from the velocity field. The magnetic field of the simulations S1b, S3b, C1b, $\mathrm{CB} 0 \mathrm{a}, \mathrm{C} 4 \mathrm{~b}$, and $\mathrm{CB} 1 \mathrm{a}$ follows a scaling very close to the isotropic scaling. In simulation $\mathrm{S} 4 \mathrm{~b}$, the relation is closer to GS95 scaling, but this is a case of weak fast modes. For the velocity field, the relationship $l_{\|}=l_{\perp}$ is followed very closely in almost all the simulations. This figure verifies that the isotropic nature of fast modes is a robust feature, and it extends throughout the inertial range. It is the same for both low and high $M_{A}$, as well as high $\beta$ and low $\beta$, as long as the driving generates a significant fraction of fast modes.

Considering the fact that the cascade of energy for Alfvén and slow modes is stronger in the perpendicular
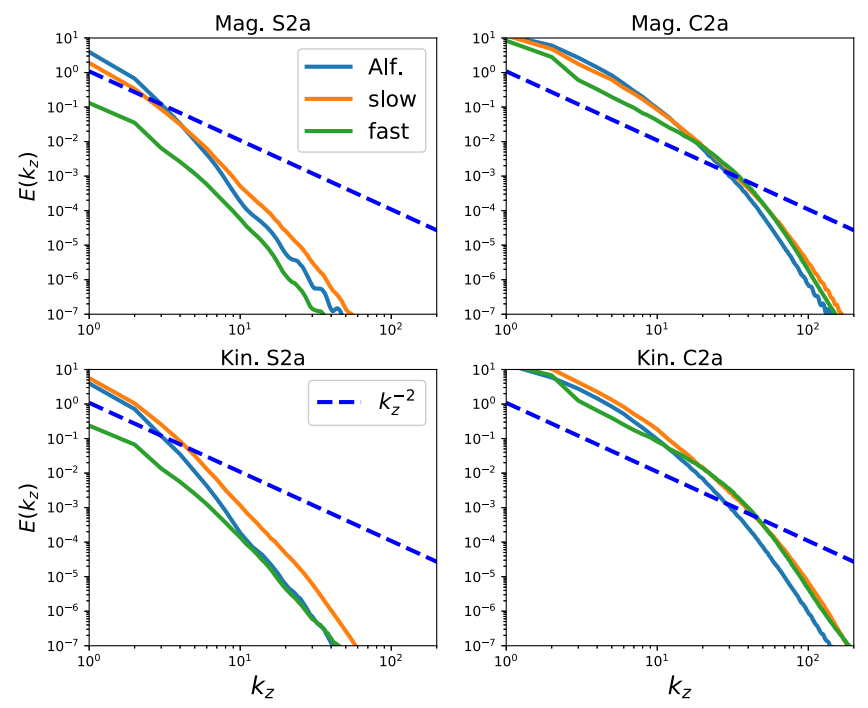

FIG. 14. The parallel wave-number spectrum for the three modes for S2a simulation (left panels) and C2a simulation (right panels), with magnetic fields (top panels) and velocity fields (bottom panels). The legends apply to all panels.

direction while for the fast mode it is isotropic, it is interesting to compare their spectrum in the parallel direction. Figure 14 compares their parallel spectra for cases S2a and C2a. In the case of S2a, the cascade of all three modes is very weak in the parallel direction as their spectra are very steep compared to the $k_{z}^{-2}$ reference slope. In the case of $\mathrm{C} 2 \mathrm{a}$, the Alfvén and slow modes are still expected to have a weak cascade. However, the fast mode will be more energetic since it is compressively driven and will be isotropic. Thus, the fast-mode parallel spectrum shows a $k_{z}^{-2}$ slope, while the Alfvén and slow modes, on the other hand, retain a weak cascade with a spectrum steeper than $k_{z}^{-2}$. As a result, even at high $k_{z}\left(k_{z}>20\right.$ in Fig. 14), the fast mode has a slightly greater energy than the Alfvén and slow modes. Thus, in this regime of compressively driven, low- $M_{A}$, weak Alfvén turbulence, the fast mode can be dominant to the Alfvén and slow modes even at large parallel wave numbers, which has implications for the cosmic-ray scattering and transport by the turbulence since the gyroresonance condition is set by the parallel wave number [20].

\section{ASTROPHYSICAL IMPLICATIONS}

This work studies the role of forcing in shaping MHD turbulence, in particular, by analyzing the decomposed linear MHD eigenmodes and their properties. The decomposition was introduced earlier to study the behavior of each eigenmode in incompressively driven turbulence [42]. We are interested in the regime of $M_{A} \lesssim 1$, where the decomposition in the Fourier space provides an effective instrument to reveal some intrinsic properties of MHD turbulence. By default, turbulence enters the MHD regime 
only when $M_{A} \lesssim 1$. Super-Alfvénic turbulence is hydrodynamic down to the scale $L / M_{A}^{3}$, where $\delta v / v_{A}$ reaches unity. Validity of the mode decomposition is further confirmed here by the distinctive 3D characteristics of each eigenmode observed in different turbulence data cubes, resulting from both solenoidally and compressively driven MHD simulations. Several observational studies also show that subsonic or transsonic turbulence is common in astrophysical plasmas. Turbulence in the HII ionized gas of Orion nebula has been observed to be subsonic [46]. Measurements of cold and dense gas in an infrared dark cloud (IRDC) have also shown many regions of subsonic Mach number turbulence [47]. Observations of the hot gaseous atmosphere of a nearby elliptical galaxy have also shown subsonic Mach number turbulence [48].

The simulations in this work use a form of forcing that can be easily decomposed into solenoidal and compressive parts. Any continuous, smooth forcing field confined in a limited region of space can always be Helmholtz decomposed into solenoidal and irrotational fields. In this sense, the forcing considered here is general. Supernova shocks and jets would drive turbulence compressively. On the other hand, magneto-rotational instability and shear-driven turbulence are probably solenoidal. There could also be mixed regions of turbulence in astrophysical jets, with the shear flows that develop between their inner and outer parts driving solenoidal modes, and the bow shock at the tip of the jet driving compressive modes [4]. Thus, one can imagine different neighboring regions of space with different types of driving and turbulence.

We find that the OU forcing provides a more realistic forcing on large scales, which can also reproduce weak turbulence features. An important demonstration of this work is the transition from weak to strong Alfvénic turbulence. This transition implies that even if the turbulence is weak at injection scales, at scales smaller than $L_{\text {inj }} M_{A}^{2}$, turbulence will transition to a strong regime of Alfvénic turbulence, which shows the Goldreich-Sridhar anisotropy. This case is important for the turbulent reconnection scenario proposed in Ref. [49]. Turbulence is closely connected with magnetic reconnection. The plasmoid instability can lead to a faster rate of reconnection [50,51]. Mean field modeling shows that a balance between turbulence-driven transport enhancement and suppression can also lead to fast reconnection [52,53]. In the turbulence reconnection scenario of Ref. [49], the transverse Alfvénmode perturbations cause field-line wandering, which modifies the Sweet-Parker reconnection rate by a factor of $\left(L / l_{\|}\right)^{1 / 2}$, where $L$ is the total length of the reconnection layer along the magnetic field while $l_{\|}$is the parallel length of a turbulent eddy. Strong Alfvénic turbulence will generate smaller lengths $l_{\|}$according to the GS95 scaling, and this result will reduce the size of the local reconnection zone, increasing the speed of reconnection. On the other hand, weak Alfvénic turbulence will not generate smaller $l_{\|}$ or speed up the reconnection rate. If the Alfvén mode is dominant, then below the transition scale, we will always have strong turbulence and hence fast turbulent reconnection, as a result. The magnetic field fluctuations in compressible modes are mostly in the parallel direction. Theoretically, this situation will reduce the field-line wandering and might not produce as much fast reconnection as in Alfvén turbulence if compressible modes dominate in turbulence. In reality, the decrease is limited since the energy fraction in Alfvén modes does not vary beyond a factor of 2 (see Fig. 2).

Turbulence leads to transport and diffusion of cosmic rays. The different turbulence modes have different scattering and transport properties of cosmic rays [20,21,54]. The compressible modes are demonstrated to dominate the transport and acceleration in turbulence. The scattering by Alfvénic turbulence is substantially reduced due to the scaledependent anisotropy. A natural consequence of having different types of turbulence in different regions of space would be an inhomogeneous transport and diffusion of cosmic rays. In this work, we have identified a regime of low- $M_{A}$ compressively driven turbulence where the isotropic fast mode can dominate over the Alfvén mode in the $k_{z}$ spectrum, which is the relevant one for gyroresonance of particles $\left(\omega-k_{z} v_{\|}=n \Omega\right.$, with $\Omega$ the gyrofrequency). Though appearing to be weak, this turbulence could still scatter cosmic rays efficiently due to the large proportion of fast modes. Studying this regime of turbulence and its implications for cosmic-ray distribution is thus important.

In the regime of strong Alfvénic turbulence, diffusion of particles in turbulent magnetic fields follows superdiffusion at scales smaller than the injection scales, $l_{\perp}^{2} \sim s^{3}$, where $l_{\perp}$ is the average separation between particles in the perpendicular direction, while $s$ is the distance traversed along the magnetic field lines $[55,56]$. This superdiffusion is because particles travel along magnetic field lines, and the field separation grows as the $3 / 2$ power of the distance traveled along field lines. This superdiffusion behavior will last until the Alfvén turbulence remains strong and the particles do not scatter. From this work, it can be confirmed that in the case of $M_{A} \lesssim 1$ turbulence, particle trajectories will separate superdiffusively at small scales until they reach the transition scale $L_{\text {inj }} M_{A}^{2}$. From then onwards, they will be in a normal diffusion regime. A larger fraction of the compressible modes in the turbulence can change the fieldline separation rate and also the mean free path of particles, which will result in a slower superdiffusion.

\section{SUMMARY}

We have analyzed the properties of the different MHD modes in solenoidally and compressively driven turbulence, which is highly relevant for astrophysical plasmas. One of the important aims of studying different MHD modes is for their different properties in particle acceleration and scattering, so these modes have been studied 
separately in this context. But important open questions are what the relative fractions of these modes are in astrophysical plasma turbulence and what they depend on.

Here, we found that the type of driving is a major factor affecting the composition of these modes. Understandably, compressive driving leads to a larger proportion of the slow-plus-fast magnetosonic modes-more specifically, the fast magnetosonic modes. Moreover, the compressive modes can also dominate in the magnetic field fluctuations. This case is crucial for the cosmic-ray transport and acceleration in turbulence since fast modes dominate the particle scattering [20,21]. A signature from polarization analysis (SPA) method to identify such modes from synchrotron polarization maps has been invented, and this study holds important implications for such measurements [57].

The nature of the turbulent cascade is also a highly relevant feature for a variety of astrophysical phenomena. We identify many important properties of the Alfvén- and slow-mode turbulent cascade from these simulations. We see the anisotropic nature of the cascade as predicted by Goldreich-Sridhar theory. We also see indications of a weak regime of Alfvénic turbulence that extends from $L_{\text {inj }}$ up to $L_{\text {inj }} M_{A}^{2}$. This is an important numerical test of a theoretically predicted regime.

On the other hand, the isotropic nature of the fast-mode cascade, which was seen earlier in low resolution studies, is now verified to extend throughout the inertial range through higher-resolution studies. This characteristic seems to be a robust feature, which does not show a weak-strong transition, unlike the Alfvén and slow modes, and does not depend on plasma $\beta$ or $M_{A}$. The spectrum of the fast modes can be steeper than $k^{-3 / 2}$ and closer to $k^{-2}$ when the fast mode dominates. This result has implications for the cutoff scale and damping of fast modes.

We argue that the mode decomposition analysis will be relevant in regions of astrophysical plasmas smaller than the driving scales of turbulence with $M_{A} \lesssim 1$ and devoid of strong gradients. This analysis implies the wide applicability of fast turbulent reconnection and superdiffusion of particle trajectories below the transition scale to strong Alfvénic turbulence. Differently driven turbulence regions will lead to inhomogeneity in particle transport and acceleration. In the case of low- $M_{A}$, compressively driven turbulence, the energy in fast modes becomes dominant even on small parallel scales. Thus, cosmic-ray scattering and acceleration will remain effective counterintuitively in this regime through both the gyroresonance and transit-time damping interactions with fast modes.

\section{ACKNOWLEDGMENTS}

The authors acknowledge the North-German Supercomputing Alliance (HLRN) for providing HPC resources that have contributed to the research results reported in this paper.
[1] R. Bruno and V. Carbone, The Solar Wind as a Turbulence Laboratory, Living Rev. Solar Phys. 10, 2 (2013).

[2] M.-M. M. Low and R. Klessen, Control of Star Formation by Supersonic Turbulence, Rev. Mod. Phys. 76, 125 (2004).

[3] P. Padoan, C. Federrath, G. Chabrier, I. Evans, N. J., D. Johnstone, J. K. Jørgensen, C. F. McKee, and Å. Nordlund, The Star Formation Rate of Molecular Clouds, in Protostars and Planets VI (University of Arizona Press, 2014), p. 77, ISBN-10: 0816531242 ISBN-13: 978-0816531240.

[4] C. Federrath, The Turbulent Formation of Stars, Phys. Today 71, No. 6, 38 (2018).

[5] R. Schlickeiser, Cosmic Ray Astrophysics (Berlin, Springer, 2002).

[6] H. Alfvén, Existence of Electromagnetic-Hydrodynamic Waves, Nature (London) 150, 405 (1942).

[7] D. G. Swanson, Plasma Waves (Institute of Physics Publishing, Bristol and Philadelphia, 2003).

[8] J. W. Belcher and L. Davis, Jr., Large-Amplitude Alfvén Waves in the Interplanetary Medium, J. Geophys. Res. 76, 3534 (1971).

[9] P. Goldreich and S. Sridhar, Toward a Theory of Interstellar Turbulence. II. Strong Alfvénic Turbulence, Astrophys. J. 438, 763 (1995).

[10] J. V. Shebalin, W. H. Matthaeus, and D. Montgomery, Anisotropy in MHD Turbulence Due to a Mean Magnetic Field, J. Plasma Phys. 29, 525 (1983).

[11] J. W. Armstrong, B. J. Rickett, and S. R. Spangler, Electron Density Power Spectrum in the Local Interstellar Medium, Astrophys. J. 443, 209 (1995).

[12] R. B. Larson, Turbulence and Star Formation in Molecular Clouds, Mon. Not. R. Astron. Soc. 194, 809 (1981).

[13] J. Roman-Duval, C. Federrath, C. Brunt, M. Heyer, J. Jackson, and R.S. Klessen, The Turbulence Spectrum of Molecular Clouds in the Galactic Ring Survey: A DensityDependent Principal Component Analysis Calibration, Astrophys. J. 740, 120 (2011).

[14] C. Federrath, J. M. Rathborne, S. N. Longmore, J. M. D. Kruijssen, J. Bally, Y. Contreras, R. M. Crocker, G. Garay, J. M. Jackson, L. Testi, and A. J. Walsh, The Link between Turbulence, Magnetic Fields, Filaments, and Star Formation in the Central Molecular Zone Cloud G0.253 + 0.016, Astrophys. J. 832, 143 (2016).

[15] J. G. Vestuto, E. C. Ostriker, and J.M. Stone, Spectral Properties of Compressible Magnetohydrodynamic Turbulence from Numerical Simulations, Astrophys. J. 590, 858 (2003).

[16] C. Federrath, On the Universality of Supersonic Turbulence, Mon. Not. R. Astron. Soc. 436, 1245 (2013).

[17] P. Grete, B. W. O'Shea, K. Beckwith, W. Schmidt, and A. Christlieb, Energy Transfer in Compressible Magnetohydrodynamic Turbulence, Phys. Plasmas 24, 092311 (2017).

[18] Y. Lithwick and P. Goldreich, Compressible Magnetohydrodynamic Turbulence in Interstellar Plasmas, Astrophys. J. 562, 279 (2001).

[19] J. Cho and A. Lazarian, Compressible Magnetohydrodynamic Turbulence: Mode Coupling, Scaling Relations, Anisotropy, Viscosity-Damped Regime and Astrophysical Implications, Mon. Not. R. Astron. Soc. 345, 325 (2003). 
[20] H. Yan and A. Lazarian, Scattering of Cosmic Rays by Magnetohydrodynamic Interstellar Turbulence, Phys. Rev. Lett. 89, 281102 (2002).

[21] H. Yan and A. Lazarian, Cosmic-Ray Scattering and Streaming in Compressible Magnetohydrodynamic Turbulence, Astrophys. J. 614, 757 (2004).

[22] L. Yang, L. Zhang, J. He, C. Tu, S. Li, X. Wang, and L. Wang, Coexistence of Slow-Mode and Alfvén-Mode Waves and Structures in 3D Compressive MHD Turbulence, Astrophys. J. 866, 41 (2018).

[23] G. Kowal, A. Lazarian, and A. Beresnyak, Density Fluctuations in MHD Turbulence: Spectra, Intermittency, and Topology, Astrophys. J. 658, 423 (2007).

[24] N. Andrés, P. C. di Leoni, P. D. Mininni, P. Dmitruk, F. Sahraoui, and W. H. Matthaeus, Interplay between Alfvén and Magnetosonic Waves in Compressible Magnetohydrodynamics Turbulence, Phys. Plasmas 24, 102314 (2017).

[25] Y. Yang, Y. Shi, M. Wan, W. H. Matthaeus, and S. Chen, Energy Cascade and Its Locality in Compressible Magnetohydrodynamic Turbulence, Phys. Rev. E 93, 061102(R) (2016).

[26] C. Federrath, J. Roman-Duval, R. S. Klessen, W. Schmidt, and M. M. M. Low, Comparing the Statistics of Interstellar Turbulence in Simulations and Observations. Solenoidal versus Compressive Turbulence Forcing, Astron. Astrophys. 512, A81 (2010).

[27] R. Meyrand, S. Galtier, and K. H. Kiyani, Direct Evidence of the Transition from Weak to Strong Magnetohydrodynamic Turbulence, Phys. Rev. Lett. 116, 105002 (2016).

[28] A. Mignone, G. Bodo, S. Massaglia, T. Matsakos, O. Tesileanu, C. Zanni, and A. Ferrari, PLUTO: A Numerical Code for Computational Astrophysics, Astrophys. J. Suppl. Ser. 170, 228 (2007).

[29] A. Mignone, A Simple and Accurate Riemann Solver for Isothermal MHD, J. Comp. Phys. 225, 1427 (2007).

[30] N. K. Yamaleev and M. H. Carpenter, Third-Order Energy Stable WENO Scheme, J. Comp. Phys. 228, 3025 (2009).

[31] V. Eswaran and S. B. Pope, An Examination of Forcing in Direct Numerical Simulations of Turbulence, Comput. Fluids 16, 257 (1988).

[32] C. Federrath, R. S. Klessen, and W. Schmidt, The Density Probability Distribution in Compressible Isothermal Turbulence: Solenoidal versus Compressive Forcing, Astrophs. J. Lett. 688, L79 (2008).

[33] W. Schmidt, W. Hillebrandt, and J. C. Niemeyer, Numerical Dissipation and the Bottleneck Effect in Simulations of Compressible Isotropic Turbulence, Comput. Fluids 35, 353 (2006).

[34] D. Told, J. Cookmeyer, F. Muller, P. Astfalk, and F. Jenko, Comparative Study of Gyrokinetic, Hybrid-Kinetic and Fully Kinetic Wave Physics for Space Plasmas, New J. Phys. 18, 065011 (2016).

[35] K. G. Klein, G. G. Howes, J. M. TenBarge, S. D. Bale, C. H. K. Chen, and C. S. Salem, Using Synthetic Spacecraft Data to Interpret Compressible Fluctuations in Solar Wind Turbulence, Astrophys. J. 755, 159 (2012).

[36] M. J. Shi, C. J. Xiao, Q. S. Li, H. G. Wang, X. G. Wang, and $\mathrm{H}$. Li, Observations of Alfvén and Slow Waves in the Solar Wind Near 1 AU, Astrophys. J. 815, 122 (2015).
[37] D. Falceta-Gonçalves, G. Kowal, E. Falgarone, and A. C.-L. Chian, Turbulence in the Interstellar Medium, Nonlinear Processes Geophys. 21, 587 (2014).

[38] D. A. Roberts and M. L. Goldstein, Spectral Signatures of Jumps and Turbulence in Interplanetary Speed and Magnetic Field Data, J. Geophys. Res. 92, 10105 (1987).

[39] A. A. Schekochihin, A. V. Nazarenko, and T. A. Yousef, Weak Alfvén-Wave Turbulence Revisited, Phys. Rev. E 85, 036406 (2012).

[40] A. Brandenburg, The Inverse Cascade and Nonlinear Alpha-Effect in Simulations of Isotropic Helical Hydromagnetic Turbulence, Astrophys. J. 550, 824 (2001).

[41] A. Brandenburg and W. Dobler, Large Scale Dynamos with Helicity Loss through Boundaries, Astron. Astrophys. 369, 329 (2001).

[42] J. Cho and A. Lazarian, Compressible Sub-Alfvénic MHD Turbulence in Low- $\beta$ Plasmas, Phys. Rev. Lett. 88, 245001 (2002).

[43] H. Yan and A. Lazarian, Cosmic-Ray Propagation: Nonlinear Diffusion Parallel and Perpendicular to Mean Magnetic Field, Astrophys. J. 673, 942 (2008).

[44] S. Boldyrev and J. C. Perez, Spectrum of Weak Magnetohydrodynamic Turbulence, Phys. Rev. Lett. 103, 225001 (2009).

[45] R. Kulsrud, Plasma Physics for Astrophysics (Princeton University, Princeton, NJ, 2005).

[46] S. J. Arthur, S.-N. X. Medina, and W. J. Henney, Turbulence in the Ionized Gas of the Orion Nebula, Mon. Not. R. Astron. Soc. 463, 2864 (2016).

[47] V. Sokolov, K. Wang, J. E. Pineda, P. Caselli, J. D. Henshaw, A. T. Barnes, J. C. Tan, F. Fontani, I. Jiménez-Serra, and Q. Zhang, Subsonic Islands within a High-Mass StarForming Infrared Dark Cloud, Astron. Astrophys. 611, L3 (2018).

[48] A. Ogorzalek, I. Zhuravleva, S. W. Allen, C. Pinto, N. Werner, A. B. Mantz, R. E. A. Canning, A. C. Fabian, J. S. Kaastra, and J. de Plaa, Improved Measurements of Turbulence in the Hot Gaseous Atmospheres of Nearby Giant Elliptical Galaxies, Mon. Not. R. Astron. Soc. 472, 1659 (2017).

[49] A. Lazarian and E. T. Vishniac, Reconnection in a Weakly Stochastic Field, Astrophys. J. 517, 700 (1999).

[50] A. Bhattacharjee, Y.-M. Huang, H. Yang, and B. Rogers, Fast Reconnection in High-Lundquist-Number Plasmas Due to the Plasmoid Instability, Phys. Plasmas 16, 112102 (2009).

[51] Y.-M. Huang, L. Comisso, and A. Bhattacharjee, Plasmoid Instability in Evolving Current Sheets and Onset of Fast Reconnection, Astrophys. J. 849, 75 (2017).

[52] N. Yokoi, K. Higashimori, and M. Hoshino, Transport Enhancement and Suppression in Turbulent Magnetic Reconnection: A Self-Consistent Turbulence Model, Phys. Plasmas 20, 122310 (2013).

[53] F. Widmer, J. Büchner, and N. Yokoi, Analysis of Fast Turbulent Reconnection with Self-Consistent Determination of Turbulence Timescale, Phys. Plasmas 26, 102112 (2019).

[54] J. W. Lynn, E. Quataert, B. D. Chandran, and I. J. Parrish, Acceleration of Relativistic Electrons by Magnetohydrodynamic Turbulence: Implications for Non-thermal Emission from Black Hole Accretion Disks, Astrophys. J. 791, 71 (2014). 
[55] L. F. Richardson, Atmospheric Diffusion Shown on a Distance-Neighbour Graph, Proc. R. Soc. A 110, 709 (1926), https://www.jstor.org/stable/94463?seq=1.

[56] A. Lazarian and H. Yan, Superdiffusion of Cosmic Rays: Implications for Cosmic Ray Acceleration, Astrophys. J. 784, 38 (2014).
[57] H. Zhang, A. Chepurnov, H. Yan, K. Makwana, R. Santos-Lima, and S. Appleby, Identification of Plasma Modes in Galactic Turbulence with Synchrotron Polarization, Nat. Astron. (2020) https://doi.org/10.1038/s41550020-1093-4 